Portland State University

PDXScholar

$1-1-2010$

\title{
Biomechanical Assessment of Parkinson's Disease
}

Edward A. Katz

Portland State University

Follow this and additional works at: https://pdxscholar.library.pdx.edu/open_access_etds Let us know how access to this document benefits you.

\section{Recommended Citation}

Katz, Edward A., "Biomechanical Assessment of Parkinson's Disease" (2010). Dissertations and Theses. Paper 83.

https://doi.org/10.15760/etd.83

This Thesis is brought to you for free and open access. It has been accepted for inclusion in Dissertations and Theses by an authorized administrator of PDXScholar. Please contact us if we can make this document more accessible: pdxscholar@pdx.edu. 
Biomechanical Assessment of Parkinson's Disease

by

Edward A. Katz

A thesis submitted in partial fulfillment of the requirements for the degree of

Master of Science

in

Electrical and Computer Engineering

Thesis Committee:

James McNames, Chair

Douglas V. Hall

Christof Teuscher

Portland State University

2010 


\begin{abstract}
Parkinson's disease is a chronic neurological disorder affecting hundreds of thousands of Americans. The current best practice for assessment of this disease is a clinical examination and subjective rating using the Unified Parkinson's Disease Rating Scale. Such ratings are coarse scaled, subject to rater bias, and costly. Instruments which provide objective measurements of disease state can eliminate rater bias, provide repeatable data, and increase the frequency and responsiveness of subject assessments, expediting the validation of new therapies and treatments.

This thesis describes the design and implementation of a battery of bio-mechanical devices suitable for clinical and in home use, including descriptions of the instruments and the functionality of the data acquisition software, as well as the overall system used for data collection. A data analysis algorithm is fully described, and descriptive statistics of pilot data from twenty two subjects are reported.

These statistics show promising correlations of time duration metrics with the motor subsection of the UPDRS, as well as good responsiveness to dopaminergic intervention. Data also suggests that these devices have an advantage over previously described devices in the ability to record the full
\end{abstract}


range of motion in standard assessment tasks, thereby providing additional metrics related to hesitations and halts in prescribed movements. 


\section{Acknowledgments}

The author would like to gratefully acknowledge the tireless contributions of the biomedical signal processing lab coordinator Brent Casady and the design team of Josh Booren and Forest Kernan, without whom this work would not be possible. Tim Albers and Dr. Lars Holmstrom made invaluable contributions to this project. Heartfelt thank are also due to Dr. James McNames, Craig Kinnie, and the Movement Disorders Clinic at the Oregon Health Sciences University neurology department.

This research was funded in part by a grant from the Kinetics Foundation. 


\section{Contents}

Abstract $\quad$ i

Acknowledgments $\quad$ iii

List of Tables $\quad$ ix

List of Figures $\quad x$

1 Introduction $\quad 1$

1.1 Parkinson's Disease . . . . . . . . . . . . . . 1

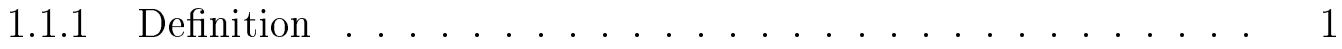

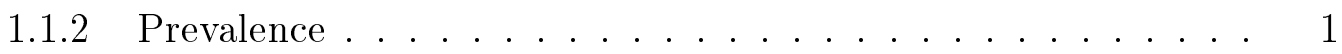

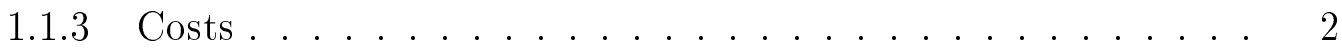

1.1.4 Primary Symptoms of Parkinsonism . . . . . . . . . . 2

1.1.5 Medications ...................... 4

1.1.6 Diagnosis ..................... 5

1.1.7 Assessment ....................... 6

1.2 Problem Statement . . . . . . . . . . . . . 8

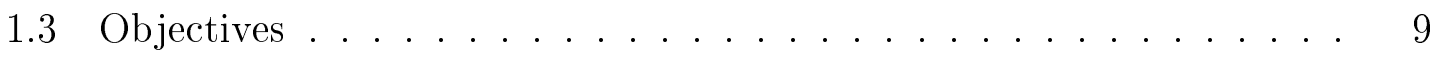

1.4 Significance . . . . . . . . . . . . . . . . 9

1.5 Demonstration of Mastery . . . . . . . . . . . . . 10 
2.1 Instrumented Tests for Quantitative Assessment . . . . . . . . . . 11

2.1.1 Button Tests ..................... 11

2.1.2 Finger Tapping Tests . . . . . . . . . . . . . 12

2.1.3 Tracking Tests . . . . . . . . . . . . . . 13

2.1.4 Purdue Pegboard Test ............... 15

2.1.5 Repetitive Alternating Finger Tapping Tests . . . . . . . . . 16

2.1.6 Spiral Tracing Tasks ............... 18

2.2 Other Instrumentation . . . . . . . . . . . . . . . . 19

2.2.1 Electromagnetic Detection ............. 19

2.2.2 Accelerometry .................... 21

2.2.3 Video Motion Capture ............... 23

2.2.4 Mounted Transducers ................ 24

3 Development of Requirements $\quad 26$

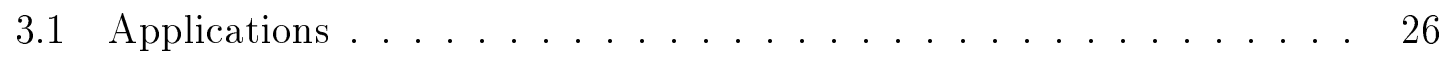

3.1.1 Clinical Trial Use . . . . . . . . . . . . 26

3.1 .2 Clinical Therapy Use . . . . . . . . . . . 27

3.2 Other Requirements . . . . . . . . . . . . . 28

3.2.1 Enforcement of Repeatability ............. 28

3.2.2 Repression of the Learning Effect . . . . . . . . . . . 29 
3.3 Summary of Requirements . . . . . . . . . . . . . . . . . . 29

4 Design $\quad 31$

4.1 Hardware . . . . . . . . . . . . . . . . . . 32

$4.1 .1 \quad$ Finger Tapper . . . . . . . . . . . . . . . . . . 33

$4.1 .2 \quad$ Foot Tapper . . . . . . . . . . . . . . . . . . . 35

4.1 .3 Transducers . . . . . . . . . . . . . . . . . 37

4.1.4 Data Acquisition Module . . . . . . . . . . . . . . . 39

4.1 .5 Notebook Computer . . . . . . . . . . . . . . . . . . 39

4.1 .6 Headphones . . . . . . . . . . . . . . . . . . . . . 40

4.2 Data Acquisition Software \& Graphical User Interface . . . . . . . 40

4.2 .1 Opening Screen . . . . . . . . . . . . . . . 40

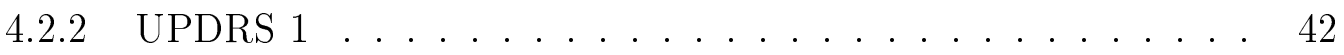

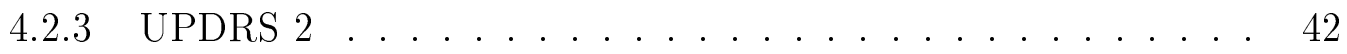

4.2 .4 Fitting the Device . . . . . . . . . . . . . . 43

4.2 .5 Initialize Position . . . . . . . . . . . . . . . 44

4.2 .6 Practice with the Bar . . . . . . . . . . . . . . 44

4.2 .7 "Fastest" Instructions . . . . . . . . . . . . . . . 45

$4.2 .8 \quad 3$ Trials Fast Test . . . . . . . . . . . . . . . . 45

4.2 .9 Headphones On . . . . . . . . . . . . . . . 47

4.2 .10 Paced Instructions . . . . . . . . . . . . . . . . . 47 
4.2.11 Paced Tapping Test . . . . . . . . . . . . . . . 48

4.2.12 Repeat Paced Tapping Test . . . . . . . . . . . . . . . 48

4.2 .13 Repeat UPDRS $1 \& 2 \ldots \ldots . \ldots . \ldots 49$

4.2 .14 Switch Devices . . . . . . . . . . . . . 49

4.2 .15 Upload Video . . . . . . . . . . . . . . . . . 49

4.2.16 The "Close" Button ................. 50

4.3 Data Analysis Software . . . . . . . . . . . . . 50

4.3.1 Populating the Data Structure ........... 50

4.3.2 Data Processing .................. 53

5 Methodology $\quad 57$

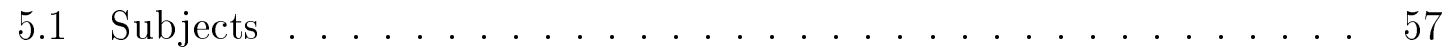

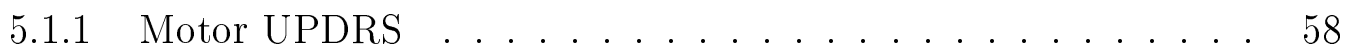

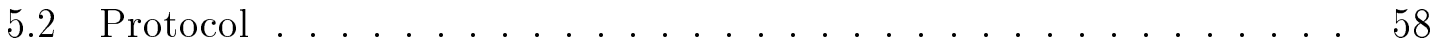

5.2 .1 Subject Information .................. 59

5.2 .2 Video Recording . . . . . . . . . . . 59

5.2.3 Three Trials Fast Test . . . . . . . . . . . . 59

5.2.4 Paced Tapping Test ................ 60

5.3 Metrics ............................. 60

5.3.1 Amplitude and Period Metrics ............ 61

5.3 .2 Event Duration Metrics .............. 61 


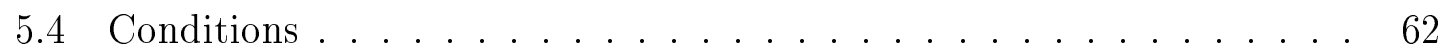

5.5 Correlation Coefficients . . . . . . . . . . . . 63

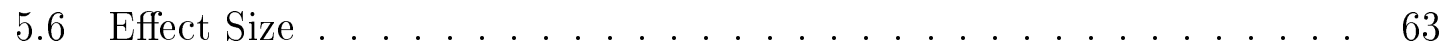

6 Results \& Discussion $\quad 65$

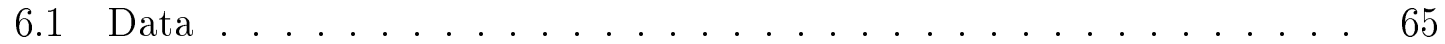

6.1.1 Group Statistics .................... 65

6.1.2 Amplitude and Period Metric Data .......... 66

6.1.3 Event Duration Metric Data ........... 67

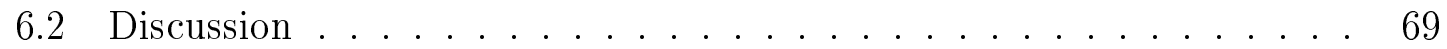

6.2.1 Clinical Notes and Test Irregularities . . . . . . . . . 69

6.2.2 Amplitude, Period, and Event Duration Metrics . . . . . . 70

6.2.3 Effect Sizes ...................... 75

$\begin{array}{lll}7 & \text { Summary \& Conclusion } & 78\end{array}$

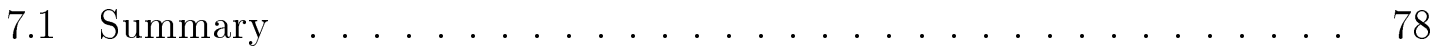

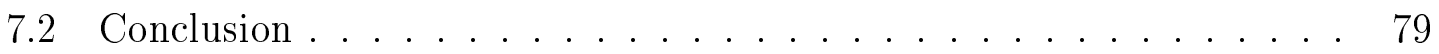

7.3 Avenues for Further Research _. . . . . . . . . . . . . . 79

$\begin{array}{ll}\text { References } & 82\end{array}$ 


\section{List of Tables}

1 Summary of prioritized goals and requirements. . . . . . . . . . 30

2 Subject information interface. . . . . . . . . . . . 41

3 Subject Cues for 3 Trials Fast Test. . . . . . . . . . . . . 46

4 Data Fields in Unprocessed .mat Files. . . . . . . . . . . . . . . 51

5 Data Structure Fields Assigned Directly. . . . . . . . . . . . . 52

6 Descriptions of event classifications. . . . . . . . . . . . 54

7 "eventStructures" Substructure Fields. . . . . . . . . . . . 56

8 Data Structure Fields Assigned to Trials in Data Processing. . . . . . 56

9 Protocol steps for each device. . . . . . . . . . . . . 58

10 List of amplitude and period metrics. . . . . . . . . . . . . 61

11 List of event duration metrics. . . . . . . . . . . . . 62

12 Conditions plotted for each metric. . . . . . . . . . 62

13 Number of subject responses. . . . . . . . . . . . . . 65

14 Correlation coefficients for amplitude and period metrics. . . . . . . 66

15 On versus off effect size for amplitude and period metrics. $\quad$. . . . . . 67

16 Correlation coefficients for event duration metrics. . . . . . . . 68

17 On versus off effect size of event duration metrics. . . . . . . . . 68

18 Event duration and period metrics with highest correlation coefficients. 72

19 Effect size metrics with highest mean effect size. . . . . . . . 75 


\section{List of Figures}

1 Signal diagram for complete system. . . . . . . . . . . . . 32

2 Finger tapping device with parts labeled. . . . . . . . . . . . 34

3 Finger tapping device showing subject arm position. . . . . . . . 35

4 Detail of finger tapping device hand grip and encoder assembly. . . . 36

$5 \quad$ Finger tapping device handgrip showing subject hand position. . . . . 37

$6 \quad$ Foot tapping device with parts labeled. . . . . . . . . . . . . 38

7 Data acquisition software opening screen . . . . . . . . . 41

8 Capture from Finger Tapper instructional video shown during "Fitting

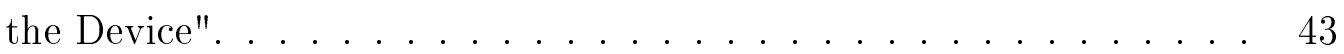

9 Capture from foot tapper practice video shown during "Practice With

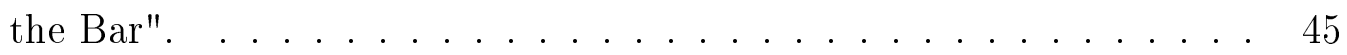

10 Block diagram of data structure population. . . . . . . . . . . 51

11 Plot of a subject trial with detected events highlighted. . . . . . . 55

12 Detail from the same trial showing trough, left saddle, peak and right

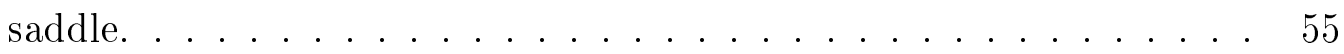

13 Detail of a trial showing a "split" trough, detected as indeterminate. . 72

14 Detail of a trial showing numerous hesitations and halts. . . . . . . 72

15 Plot of the correlation of left saddle time with respect to motor UPDRS. 73 
16 Plot of the correlation of indeterminate event time with respect to motor UPDRS ........................... 73

17 Plot of the correlation of total peak time with respect to motor UPDRS. 73

18 Plot of the correlation of mean trough period with respect to motor

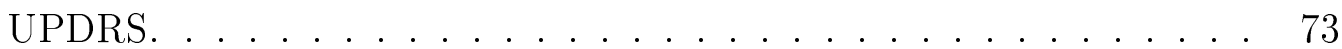

19 Plot of the correlation of indeterminate event time with respect to motor UPDRS. ......................... 74

20 Plot of the correlation of left saddle time with respect to motor UPDRS 74

21 Plot of the effect size of trough period standard deviation. $\quad \ldots . . . \quad 77$

22 Plot of the effect size of mean trough period. . . . . . . . . . 77

23 Plot of the effect size of the total number of taps. . . . . . . . . 77

24 Plot of the effect size of mean cycle amplitudes. . . . . . . . . . 77 


\section{Introduction}

\subsection{Parkinson's Disease}

\subsubsection{Definition}

Parkinson's disease is a chronic, progressive neurodegenerative disorder affecting more than 350,000 Americans. The effects of the disease are characterized by prolonged disability and steadily worsening symptoms, although it is not generally in itself fatal.

\subsubsection{Prevalence}

Parkinson's disease is the second most common neurodegenerative condition after Alzhiemers disease [5]. Estimates of total prevalence vary widely, but approximately 50,000 people are diagnosed each year, and between 350,000 and 1,000,000 people suffer from the disease at any given time $[2,4]$. Parkinson's is primarily a disease of the aged-the average onset age is 60 years, with only $5-10 \%$ of reported cases occurring in persons under 40 . It is estimated that $1.6 \%$ of persons over the age of 65 and $2.4 \%$ of persons aged 80 to 89 have some form of the disease [3-5]. The population over the age of 65 is expected to double by 2040, with a larger portion of those surviving beyond 85, thus the overall prevalence of Parkinson's disease is expected to increase as well $[3,5,6]$. 


\subsubsection{Costs}

The fiduciary cost to individuals is significant. Persons registered for Medicare pay an average of 2.5 times the out of pocket costs of other seniors [4], much of this is in prescription drugs, which average about $\$ 2500$ a year. Most of the remainder is due to "co-morbidities" such as hospitalization due to falls and institutionalization for dementia or other care needs. With office visits and incidental costs, the total outlay due to Parkinson's disease in America is estimated at $\$ 5.6$ Billion a year. Costs including lost productivity and unpaid care are estimated at $\$ 23-34$ Billion a year $[3,4]$.

\subsubsection{Primary Symptoms of Parkinsonism}

Parkinsonism is a general term for the set of symptoms associated with Parkinson's disease [2]. A number of causes and conditions that present similarly. The four cardinal symptoms of Parkinsonism are tremor, rigidity, bradykinesia, and postural instability.

Parkinsonian tremor is a resting tremor, which becomes more obvious and severe when the person is resting and improves with intentional movement $[1,2]$. Tremor tends to be present in the hands, arms, legs, jaw and face [1]. Facial tremor usually involves the jaw, tongue and facial muscles, and not the shaking of the head seen in

essential tremor [2]. Tremor in the hand is typically of the "pill rolling" type, where 
the thumb and forefingers seem to rotate about some point. Parkinsonian tremor is generally of fairly low frequency, ranging from 2.5 to $5 \mathrm{~Hz}$ [1] and is activated or increased by stress or emotional excitement. Tremor is typically not present while sleeping [2].

Rigidity is a stiff and weak feeling in the limbs and trunk. Rigidity can manifest as either a steady "lead pipe" resistance to movement, which occurs when a person's muscles remain tense and contracted, or as a "cogwheel effect", where resistance to movement occurs in short, jerky steps. This is caused by the lack of synchronization between antagonistic muscle pairs [1].

Bradykinesia is a general slowing of movement, sometimes coupled with an inability to initiate movement or akinesia. In advanced Parkinson's bradykinesia is subject to rapid fluctuations from ease of movement to inability to move, especially as medication doses wear off [2].

People with Parkinson's suffer from postural instability, in the form of impaired balance and coordination. This also manifests as a stooped and droopy posture, as well as halts and freezes while walking [1]. They also have a tendency to lean backwards or take short backward steps when bumped or starting to walk, an effect called "retropulsion". People with advanced Parkinson's tend to walk with short, rapid steps, which is called "festination" [2]. 


\subsubsection{Medications}

The biological cause of Parkinson's disease is the death of neural cells in a part of the brain called the substantia nigra. This portion of the brain produces a neurotransmitter called dopamine, which is essentially the medium by which signals are passed between neurons. The substantia nigra is the primary dopamine source for the corpus striatum, which helps regulate movement throughout the body. As the disease progresses and less dopamine is delivered to the corpus striatum outgoing signals become unreliable and movements become erratic and uncontrolled.

The primary medication used to treat Parkinson's disease is levodopa. Dopamine itself is not effective as a medication because it cannot cross the blood-brian barrier, but levodopa is a dopamine precursor that can do so. It is then metabolized into dopamine by the enzyme dopa decarboxylase [2] in the substantia nigra. Because this enzyme is found throughout the body, very large doses of levodopa would be required to be effective. To counter this, dopa decarboxylase inhibitors (DDI's) such as carbidopa or benserazide are given with the levodopa dose. These are "peripheral" DDI's, in that they inhibit the metabolization of levodopa in the bloodstream, but do not affect metabolization in the brain.

In the United States, the most common medication is sinemet, a carbidopa/levodopa formulation available in various dossages and proportions. As the disease progresses and neurons in the substantia nigra die, the brain's capacity to metabolize levodopa 
decreases and the medication dose must be increased. This is not a tolerance or loss of potency of the medication, but an effect of advancing degeneration [1] [2].

These medications can have unwanted side effects. The most noticeable side effect of levodopa is dyskinesia, or uncontrolled, swaying movements. Large, dance like movements called choreiform dyskinesias are seen in advanced patients. These movements typically occur at the peak of a leveodopa dose, although they also occasionally occur at the beginning and end of a medication cycle $[1,2]$. Other side effects can include vivid dreams and nightmares, paranoia, and walking hallucinations. Occasionally these are severe enough to require institutionalization, especially in patients with reduced mental capacity or severe dementia [2].

\subsubsection{Diagnosis}

Diagnosis of Parkinson's disease is made clinically based on the person's history and symptoms. There is no definitive laboratory test for Parkinson's disease. MRI and CAT scans do not reveal Parkinson's, but can be useful in eliminating alternate diagnosis, such as cerebrovascular disease (stroke) [2], which may present similar symptoms. Radiological imaging devices such as PET and SPECT may reveal Parkinson's, but cannot necessarily differentiate Parkinson's from other neurodegenerative conditions. In addition, the cost and complexity of these devices tends to limit them to research facilities [7]. Protein aggregates called Lewy bodies are found 
in the brain tissue in Parkinsonian patients. Unfortunately, these can only be seen in autopsy. Such structures are also found in other diseases such as Multiple Systems Atrophy(MSA) and Pick's disease.

\subsubsection{Assessment}

The current best practice for assessment of Parkinson's disease is physical examination by a trained clinician using the Unified Parkinson's Disease Rating Scale (UPDRS). The clinician assigns scores to forty two items on the UPDRS by physical examination and verbal inquiry. The scale is divided into four sections: Mentation, Behavior and Mood; Activities of Daily Living; Motor Examination and Complications of Therapy.

Each section contains a number of items which are rated on a scale of 0 to 5 by the clinician, with higher numbers correlating to greater severity of symptoms. In addition a series of "yes" or "no" questions are rated as 0 or 1.

Biomechanical assessment devices are concerned primarily with the Motor Examination section. The items in this section deal with physical observables related to movement speed and muscular control, which are in turn correlated to the cardinal symptoms of Parkinson's disease.

People with Parkinson's are also rated on the Modified Hoehn and Yahr staging scale, which rates the general level of impairment on a six step scale, with scores 
increasing as severity increases. The Schwab and England "activities of daily life" is also used to rate subject's independence and ability to perform routine tasks. It should be noted that this scale is rated from 100 to 0 , where 0 is complete helplessness; i.e. scores decrease with advancing illness.

Parkinson's patients are also often assessed for cognitive impairment, as a number of items on the UPDRS depend on their ability to understand and respond to specific queries and instructions. An example is the "Mini Mental State Examination", in which subjects are rated on a number of questions focusing on orientation, recall and language ability. This examination is also used to exclude subjects from clinical trials.

The UPDRS examination is the accepted rating scale for Parkinson's Disease progression, and in the absence of valid biomarkers, it is the prevailing standard for diagnosis and assessment of severity.The examination exhibits good intra and inter-rater reliability, and addresses a variety of symptoms beyond the "Physical" motor symptoms. Disadvantages of UPDRS examination include it's coarse rating scale, which results in in poor responsiveness to changes in disease state, and the requirement for trained personnel, which complicates blinding in studies, drives up the cost of clinical practice, and is impractical for home assessment. 


\subsection{Problem Statement}

As mentioned above, assessment of Parkinson's disease by clinicians using the UPDRS has several disadvantages. The level of training required restricts the number of raters available, making rating time scarce, and by extension ratings expensive. The scarcity of clinician time restricts the number of ratings an individual receives, reducing the number of data points in any assessment of therapy. It is also not practical to have clinical raters travel to subjects' homes, which requires subjects to travel to a clinic for assessment. The stress and activity of such a visit may confound symptoms and alter performance. Furthermore, the scarcity of clinician hours for additional ratings makes it difficult to scale up studies. In general, it is not possible to suddenly and dramatically increase the number of raters to support a new study. The reliability of the UPDRS is largely due to its coarse scale and the large number of symptoms assessed. Assessments of individual symptoms may be subject to rater bias and subjective scoring.

Biomechanical devices can overcome many of these difficulties. Devices could be issued to subjects, allowing for use at home and at leisure. Assessments could then be done every day or more, capturing medication cycles and diurnal fluctuations. In the case that large numbers of devices are manufactured for large studies, we would expect economies of scale and reduced unit costs. Furthermore, mechanical devices provide objective measures, and need not be blinded to subject identity. To 
achieve this, devices must be reliable, inexpensive, and allow for the possibility of self administered testing while providing measures that reflect the disease state of the subject.

\subsection{Objectives}

The objective of this thesis is to demonstrate a biomechanical system which provides useful metrics for the assessment of the severity of Parkinson's disease, specifically focused on bradykinesia. For the purposes of this thesis, useful will be defined as being responsive to dopaminagenic intervention, specifically as distinguishing between the on and off medication states of a group of subjects to within a certain confidence range. The system will include the electromechanical devices, the analysis algorithms that provide metrics from raw measurements, and the interface software directs the testing protocol while collecting and storing data.

\subsection{Significance}

The significance of such a device could include the possible production of a set of measurements not well described in the literature. Such a device could be suitable for clinical use, reducing demand on personnel and allowing for greater patient flow. Such clinical use could provide a standard for the validation of new therapies.

The system will be suitable for clinical trials, again providing a standard for other 
measurements and therapies. This, combined with faster assessment and greater patient throughput, could result in faster validation of assessments and interventions.

The system will also be suitable for at home use, allowing for more frequent assessments and reducing confounding factors. Again, additional data points and reduced demand on trained clinicians could expedite validation of treatments and therapies.

\subsection{Demonstration of Mastery}

This thesis is intended to demonstrate mastery of knowledge in the field of Electrical and Computer Engineering. This demonstration includes the completion of a literature survey to assess the current state of the art in a given discipline, as seen in chapter 2; the systematic creation of requirements based on use cases, demonstrated in chapter 3. Significant contributions were made to the design, implementation and testing of a device in cooperation with cross-disciplinary colleagues, and the creation of analysis software, as seen in chapters 4 and 5 . 


\section{Existing Technologies \& Literature Survey}

A number of tests and devices have been described with similar objectives to those described in chapter 1 . In this chapter some of these are discussed for the purpose of informing the requirements analysis and final design in subsequent chapters. An understanding of previous work by other researchers will highlight the advantages of the devices described in this thesis.

\subsection{Instrumented Tests for Quantitative Assessment}

\subsubsection{Button Tests}

Various authors have used button tests to quantify bradykinesia. In it's most basic form, a pair of buttons are placed approximately shoulder width apart on a table in front of the subject. When cued to begin, the subject alternately taps each button as fast as they are able. The button press times are recorded and the rhythmicity and duration of movement are then computed. Additionally, the subject may be asked to hold a button down before beginning. The subject is then cued to begin, and the time between the cue and the release of the button is recorded as "reaction time".

The test is advantageous in the simplicity of design and the ease with which data can be electronically stored. Disadvantages of devices of this type include an ongoing learning effect [38], as well as being unable to distinguish hesitations or velocity changes from long duration continuous movements. These tests are also 
subject to strategies by the test taker, such as a "speed versus accuracy" tradeoff, where a subject can increase the rate of tapping at the expense of occasionally missing a button strike.

Giovannoni et al. [23] attempt to quantify this effect using a keyboard in place of a button. A target key is defined, and strikes on the target key as well as surrounding keys are recorded. Strikes on the surrounding keys were recorded as "missed keys" and used to represent accuracy. They found that the number of missed keys increases rapidly above a certain threshold rate of keystrikes (described as the "Dysmetria Turning Point"). They were then able to create a combined score, but did not find correlation with the UPDRS. Ghika et al. [19] and Ward [28] measured reaction time and movement time with button devices, and found the movement time of people with Parkinson's to be significantly longer than that of age matched controls, while reaction time was not significant. Dunnewold, et al. [16] also found subjects had a significantly lower tap rate than controls.

\subsubsection{Finger Tapping Tests}

Finger tapping tests in general imitate the twenty-third item on the UPDRS, where the subject taps the thumb with the index finger in rapid succession. In general, more distal movements are more affected by bradykinesia, and have the potential to provide more responsive metrics. This simple task is also easily learned, and with a 
few moments practice a subject is typically as skilled at the task as they are likely to get, reducing or eliminating the learning effect. In addition, there are only minor testing strategies associated with this task, notably a "flicking" motion instead of a clamping tap.

The disadvantage of this task is the difficulty of instrumentation. Because of the "free" posture associated with this movement, devices that attach to the subject tend to restrict movement. This leads researchers to devices that can detect movement without contact and at a distance, which tend to be complex and expensive. In addition, by restricting measurement to one of the set of 42 items on the UPDRS, variations in the effect of the disease from subject to subject inevitably reduce the correlation to the UPDRS as a whole, making it more difficult to establish validity.

\subsubsection{Tracking Tests}

Tracking tests involve the subject manipulating a device in some way which is not predictable in advance. The feedback between a changing objective and perception of current position has been found to be particularly sensitive to disease state. Many of these tests are computer based, taking advantage of available input devices, as well as the ease of data storage and change in instructions. Like many of the previously described tests, tracking tests are often subject to the learning effect and speed versus accuracy strategies. 
Allen et al. [20] explored the use of video game peripherals, specifically a joystick and a steering wheel, to implement tracking tests on a computer. Subjects were asked to use the peripheral input device to follow a moving target on the screen for four different protocols: pseudo random movement, swept frequency, alternating between fixed points, and alternating between fixed points with a trigger button. The first two are standard tracking tasks, while the second two imitate tapping tests similar to the button tests mentioned above. They found the best separation between people with Parkinson's and controls for the fixed-point targets.

Montgomery et al. [40] developed a wrist flexion-extension device consisting of a conical receptical into which the subjects hand is inserted. The device allows the subject to flex and extend the wrist, pointing the hand at LED targets arranged in an arc. The two rows of LED targets represent the position of the hand and the target position. When a target light is lit, the subject moves his hand until the position LED corresponds to the target. By recording movement time as well as target time, reaction time could be computed. By setting targets near to but not on the end of the target row, overshoot could be measured. By setting targets in pseudo-random patterns, subject tracking ability could be tested. They did not find this test in itself to be particularly responsive to early stage Parkinson's disease, although combined with olfactory and mood-mentation tests the combined result was statistically significant. 


\subsubsection{Purdue Pegboard Test}

The Purdue pegboard test was developed in 1948 as a dexterity test for personnel selection [10], but has emerged as a sensitive assessment of Parkinson's disease [11]. In the standardized version of the test, a board with two vertical rows of 25 holes each is placed on a table before the subject. On either side of the rows of holes are cups containing 25 pegs. The subject is instructed to place as many pegs in holes in 30 seconds as possible, first with the right hand, then with the left, then with both hands simultaneously. Some versions of the test have an additional "assembly" task, where washers and caps are placed on the pegs [9].

The test exhibits good to excellent test-retest reliability $[9,10]$ and very good correlation to the UPDRS, but poor distinction between symptoms. Pegboard scores suffer with advancing bradykinesia as well as tremor. In addition, a learning effect has been reported since the test's inception, and like the button test above, the test is subject to speed versus accuracy strategies. Researchers have also noted a difference in performance between men and women and between education levels [10].

Despite these drawbacks, Haaxma et al. [11] found the pegboard to be a responsive and reliable instrument, and was in fact by itself as sensitive as the combination of concurrently given tapping, writing and walking tests. 


\subsubsection{Repetitive Alternating Finger Tapping Tests}

Another tapping tasks that has been described in the literature is Repetitive Alternating Finger Tapping (RAFT). In this test the subject taps two fingers alternately as fast as possible, typically on a MIDI piano keyboard or similar device. Having recorded the duration and timing of keystrikes, the rate, rythmicity and velocity can then be computed. In some cases metronome-like audio cues are provided and the subject's ability to follow the provided rhythm is tested.

The defined MIDI standard records keystrike start and stop times which are then encoded as velocity and duration, as well as allowing for variable sample rates. Equipment is commercially available and data is digitized instantly. MIDI keyboards do not record a full range of movement; the position of the key is detected by an encoder with limited resolution. This allows for velocity computation and detection of key release, but the small physical displacement may or may not be accurately represented.

Bronte-Stewert et al. [34] instructed subjects to repetitively tap two adjacent keys with the index and middle fingers for 60 seconds, as fast and as regularly as they could. The subjects were blindfolded and white noise headphones were placed over their ears to remove visual and audible feedback. The velocity and duration of keystrikes and the interval between them was recorded, and the means and coefficients of variation computed for each. The keystrikes were also examined 
for signs of fatigue, freezing, and failure to alternate between keys. They found that while controls were able to maintain the alternating keystrikes for the full 60 seconds, Parkinsonian subjects' performance began to degrade after as little as 10 seconds. They also found occasional sections of high frequency (i.e greater than 4 $\mathrm{Hz}$ ) non-alternating tapping that could be classified as tremor.

Koop et al. [22] instructed subjects to repetitively tap two adjacent keys with the index and middle fingers for 30 seconds. The velocity of the keystrikes was then recorded and the mean velocity computed. They found that the mean velocity separated control subjects from early PD subjects, and that finger and forearm bradykinesia could be detected by this method in subjects who had not yet developed postural velocity symptoms, as seen in concurrent dynamic posturography measurements.

Tavares et al. [33] performed a similar test on subjects before and after bilateral subthalamic nucleus deep brain stimulation (B STN DBS), a neurosurgical procedure involving electrical stimulation of the subthalamic nucleus, which can be effective in controlling severe symptoms [2]. Subjects again tap two adjacent keys for 30 seconds. In this case the mean velocity, duration of finger strike and interval between strikes were computed for each finger. The coefficient of variation was then computed for each of these measures. They found that the combination of mean velocity, mean duration, and coefficient of variation of duration showed the highest correlation to the 
UPDRS motor section. Furthermore, both dopaminergic medication and B STN DBS improved the velocity of, and interval between, keystrikes, while duration, coefficient of variation of duration, and coefficient of variation of interval improved more with B STN DBS than with medication.

\subsubsection{Spiral Tracing Tasks}

A common test for the assessment of tremor and dyskinesia is the tracing over a spiral printed on a piece of paper. With the advent of digital tablets, this test became popular due to the immediate digitization of data, as well as tasks and testing protocols that can be controlled in software, making them relatively easy to adjust. The digital tablet is relatively inexpensive and intuitive to use, making it ideal for at-home studies.

The primary disadvantage of tablets is the limited movements they can record. They are essentially restricted to movements of the hand and wrist, in two dimensions, making it very difficult to imitate existing items on the UPDRS.

Liu et al.1 [15] used a digital tablet to analyze round and square spirals, traced by subjects suffering from dyskinesia induced by levodopa. They were able to correlate measurements derived from the spiral trace to UPDRS dyskinesia scores, although they did not observe a difference between square and round spirals.

Ide et al. [18] added a tracking task consisting of a screen display with a marker 
indicating the position of the pen on the tablet, and a target to which the subject is to move the pen. As the target moves and the subject attempts to follow it, the positions of the target and pen are recorded, and metrics such as reaction time, position error, and pen velocity are extracted. They found significant differences in subjects before and after deep brain stimulation.

\subsection{Other Instrumentation}

\subsubsection{Electromagnetic Detection}

Various schemes have been implemented for electromagnetic detection of position, which would then be combined with UPDRS tasks for quantification. The attraction of such a system is the freedom of movement which would allow for a variety of tasks. It is reasonable to believe that the reduction in repeatability with unrestricted movement would be offset by the additional tasks available.

Kandori et al. [12] constructed such a device to measure position during a gripand-release task, a test used by surgeons to diagnose myelopathic symptoms in a patient's hands. The system consists of a $20 \mathrm{kHz}$ oscillator feeding a transmit coil mounted on the wrist and five detection coils mounted on four fingers and the forearm. When energized by the magnetic field produced by the oscillator, the detector coils produce a voltage which is amplified and compared to the $20 \mathrm{kHz}$ oscillator signal using a phase detector. The resulting phase difference is low pass filtered and 
output to analog to digital converter for digital storage and analysis.

Healthy volunteers affixed the device to their dominant hand and gripped a $30 \mathrm{~cm}$ reference rod. They were then asked to open and close their hands as fast as they were able to for 15 seconds, repeating for the non-dominant hand. Although they were able to detect specific events (i.e. the opening and closing of the hand), Kandori et al. were not able to provide direct position information due to the non-linearity of the detected voltage.

The 3Space Fastrack (Polhemus, Colchester, VT) is a commercial electromagnetic tracking device that has been used to quantify amplitude and speed impairment, as well as tremor. The Fastrack has a centralized transmitter and up to four passive sensors. The sensors contain orthogonal coils detecting the AC magnetic field produced by the transmitter. The device then computes position and orientation with six degrees of freedom in real time. The stated range of the device is $4-6$ feet, with a static position and angular accuracy of $.0015 "$ and .15 degrees.

Espay et al. [13] affixed sensors to subjects' thumb and forefinger while they performed a tapping task, then to the forefinger and jaw for a finger-to-chin task. This study was designed around the dissociation of speed and amplitude metrics, and they found that amplitude was more affected by medication state than speed. They further found amplitude measurements to correlate more strongly to UPDRS ratings, while speed measurements were more closely related to timed tests, notably 
the Purdue Pegboard and tapping tests.

Spyers-Ashby et al. [14] used the same device to quantify tremor, in an effort to distinguish between Parkinson's disease, essential tremor and multiple sclerosis. In this case the sensor was affixed to the dorsal face of the hand and subjects were seated in a straight backed chair with their arm extended. By analysis of time domain position data they were able to distinguish between normal subjects and patients and multiple sclerosis patients from Parkinson's patients very well, but it was more difficult to distinguish between subjects with Parkinson's and those with essential tremor.

\subsubsection{Accelerometry}

Accelerometers have been used extensively by many researchers. The small size and "passive" nature (i.e. no specific movement is required for data) make them ideal for assessment of movement disorders. Accelerometric data can be taken concurrently with other tests such as button tests or the UPDRS. In addition, accelerometric data can be taken continuously, allowing for a broader assessment of motor function than is possible with discrete, "snapshot" tests.

Disadvantages of these devices include the relatively complex signal processing required to extract meaningful measurements. Furthermore, continuous measurements require continuous power and data storage, increasing the complexity and cost of the 
electronic system.

Dunnewold et al. [16] used two and three axis accelerometers in conjunction with a button test, and found two axes sufficient for the assessment of bradykinesia. They also show that resting tremor did not adversely affect their assessment. Sherrill et al. [26] affixed nine accelerometers to subjects while they performed a set of UPDRS tasks. Motor UPDRS and dyskinesia were then assessed by video review. They report a correlation between features extracted from the accelerometric data and clinicians assessments.

Meyers et al. [29], affixed a uniaxial accelerometer to the index finger while surface electromyography (EMG) leads were affixed to the extensor digitorum and first dorsal interosseous muscles on the same finger. Subjects performed a tapping task where they tap the instrumented finger while attempting to synchronize to an intermittent tone with a constant rhythm, they are then asked to continue tapping at the same pace after the tone stops. The test was repeated on and off medication. They report accelerometric measurements being more sensitive to medication than the EMG signal, although both could distinguish between on and off states. In addition, they found that variability in movements tend to change over time, with internally cued movements being much more variable than those cued by the external pace tone. 


\subsubsection{Video Motion Capture}

Video motion capture can be used in conjunction with a variety of motor tasks, including finger movements such as tapping tests, as well as larger movements such as arm pronation/supination or walking gait. In general, a set of passive reflective markers are placed on the subject at points where movement is to be tracked. An infrared light source and a set of orthogonally oriented cameras with selective filters are then used to record the movement of the markers. By knowing the initial position of the markers with respect to the cameras, the movements can then be reconstructed as data, and computations performed.

The disadvantage of such systems is that they are generally large, expensive, and not practical for at home or continuous use. The signal processing involved in reconstructing movements can also be complex and difficult.

Agostino et al. [32] used a three dimensional infrared system to measure kinematic performance of subjects instructed to tap their index finger against their thumb for three five-second trials, then each of their fingers in turn against their thumb, again for three five-second trials. The motion of the finger was recorded and the number of taps, the amplitudes of extension and flexion, the durations of flexion and extension, and the pause between were computed. They found that people with Parkinson's had lower amplitudes and longer pauses than controls, and that their performance decreased more rapidly with additional trials. 
Jobbagy [39] assembled a system using infrared markers and a consumer-grade video camera. Subjects placed their hands in prescribed positions in front of the camera and tapped each finger in turn in a piano-like movement, lifting each finger as rapidly and as high as they can. The position of each finger is recorded and a "Finger Tapping Test Score" (FTTS) is composed of amplitude, frequency and periodicity is then computed. They show that the FTTS is lower in subjects with Parkinson's disease than in controls, and that subjects with Parkinson's often have substantial differences between right and left hands.

\subsubsection{Mounted Transducers}

A more direct way to capture movement is to record position directly with mechanical movement transducers. Devices using potentiometers and encoders mechanically coupled directly to the subject can be used to record the full range of simple movements with great accuracy and resolution. Advantages of such systems include relatively simple signal processing and low cost, intuitive devices.

The primary disadvantage of such systems is the difficulty of mechanically monitoring a movement without interfering with or restricting said movement.

Sauermann et al. [17] used a hand held thumb trigger with a resistive transducer to measure the rate and position of thumb tapping. By measuring the full range of motion, they were also able to directly quantify periods of hesitation or freezing, a 
bradykinetic effect that would otherwise only be seen as a component of the overall rate of tapping. They found good correlation with the 25th item on the UDPRS, hand pronation/supination. The time spent in hesitation also correlated with the hand opening and closing score, UPDRS 24.

Andria et al. [27] measured the force applied by a palmer grip. The device consists of a palm-rest and finger handle, instrumented with force cells. Subjects were cued by an audio tone to begin squeezing the handle as hard as possible for 6 seconds, at which time a tone was played to cue them to stop. The rise time, fall time, and total force applied were then computed. They found that the total force and the fall time were most relevant in differentiating between people with Parkinson's and controls. 


\section{Development of Requirements}

\subsection{Applications}

\subsubsection{Clinical Trial Use}

To develop goals and requirements, two distinct applications are considered. The first case is use in clinical trials to assist in validating new therapies where a clinician currently assesses the subject using the UPDRS. The objective in the short term is to provide a metric to augment the UPDRS, and in the long term to replace it all together with quantitative assessments.

The clinical trial case requirements are in some sense the most urgent, as participation in clinical trials would be part of the process to validate any device developed. Trials conducted in clinic allow the possibility of assistance and instructions for the subject, and the centralized location reduces the need for a mass-producible device. The physical and mechanical design must be such that it is well tolerated by clinicians and subjects. Complicated or hard to use devices are less desirable to those actually administrating the study, physical discomfort or restriction of movement is likely to reduce subject participation.

Clinical trials involving at-home use have additional requirements. The advantages of a self administered test taken at home include additional data points which may capture daily or medication cycle fluctuations and the reduction of travel and 
clinical visit related confounders mentioned in Section 1.3. An at home test would be repeated by the same subject many times with no assistance or supervision, which requires the possibility of self administration. This implies a lack of difficult straps, fitments, or restraints. Any cues or instructions must be pre-recorded or automated, and data must be recorded and reported automatically. Such devices must also be sized in such a way that subjects can reasonably be expected to accommodate them in their homes, and be portable enough to be shipped. Bringing the devices to subjects rather than subjects to centralized devices also implies that many more devices would be needed, requiring a manufacturable and inexpensive design.

\subsubsection{Clinical Therapy Use}

The second case is day-to-day clinical practice. A quantitative device could be provided to a clinician who would use it in standard assessment of disease progress. This would in general imply a greater number of subjects using the device regularly.

The design requirements for this case include all those mentioned above in clinical trials, with the additional requirement that such devices must be fully and convincingly validated as providing measures of the progression of the disease. The ease of use criteria also becomes more important in this case, as clinicians are unlikely to incorporate a new device into their practice if the inconvenience of use outweighs the benefit, and subjects are less likely to regularly use such a device at home. 


\section{$3.2 \quad$ Other Requirements}

In addition to the requirements specific to application, a set of general goals apply to all devices, specifically balancing the conflicting goals of test repeatability versus subject comfort and minimization of the learning effect.

\subsubsection{Enforcement of Repeatability}

For repeatability, it is desirable for a subject to take a test the same way each time, as should multiple subjects taking the same test. Variation can be introduced by subject conditions such as fatigue and learning, as well as test taking strategies which may be employed.

One design concept employed to reduce variability is that of "enforced compliance", or designing devices in such a way that there is only one way to take a test, so that each subject wants to do it in the same way. This is difficult in practice, as nearly all biomechanical test devices are subject to some form of strategy.

In many cases, compliance can be enforced and strategies reduced by constraining the subjects movement in some way. Although this can reduce variability, there are disadvantages to this approach. The primary disadvantage is subjects and clinicians in general do not like it. Subjects respond negatively to being "strapped in" in any way, especially if they are unable to extricate themselves under their own power. Clinicians prefer not to have complicated devices that require extra training for 
administrators and extra set up time for subjects.

\subsubsection{Repression of the Learning Effect}

To reduce the learning effect, tests should be designed to be "learned" as fast as possible, and subjects should be allowed to practice using any new device before data is taken. This can most easily be accomplished by simple, repetitive movements. Movements that are a part of everyday tasks are also desirable, on the grounds that subjects have already "practiced" these tasks on a day to day basis. Caution should be taken to avoid assuming uniform proficiency across populations however. Certain subpopulations, such as pianists or ten key operators, may exhibit greater abilities at tasks resembling their vocations.

\subsection{Summary of Requirements}

All of the requirements above are considered in the process of developing new devices. It is quite possible, however that not all of them can be realized in the short term. Furthermore, qualitative goals such as convenience and reduction of strategies can be satisfied to varying degrees. The design goals and requirements are assigned priorities in Table 1. Each priority is rated on a scale of 1 to 5 , where 5 is absolutely required for any degree of success, 4 is greatly desired, and others are prioritized in numerical order. 
Goal

Priority

\begin{tabular}{ll}
\hline \hline Tolerance by subject & 5 \\
Convenience of use for clinician & 5 \\
Minimization of learning effect & 4 \\
Minimization of strategies & 4 \\
Minimal restriction of movement & 3 \\
No attachment to subject & 4 \\
Can be self administered (mechanically) & 4 \\
Can be self administered (software) & 3 \\
Automatic data upload (software) & 3 \\
Portable & 2 \\
Can be accommodated in home & 3 \\
Mechanically robust & 2 \\
Manufacturable Design & 2 \\
Low cost design & 1 \\
\hline
\end{tabular}

Table 1: Summary of prioritized goals and requirements. 


\section{Design}

This chapter provides technical descriptions and design justifications for the devices in the Biomedical Signal Processing (BSP) lab at PSU, with which pilot data was collected at the Movement Disorders Clinic at the Oregon Health Sciences University (OHSU) neurology department. This includes the trigger-type finger tapper and the foot tapper, as well as the software, protocol, PC and data acquisition device used to collect the data in chapter 6 . The biomechanical devices, data acquisition, and PC are described in Section 4.1. The set of tests and instructions are referred to collectively as the Protocol, described in Section 5.2, while the MATLAB script that provides instructions and records data is referred to as the Data Acquisition Software, described in Section 4.2. The data collected is analyzed by the data analysis software, described in Section 4.3.

Figure 1 below describes the overall layout of the system. Each biomechanical device is fitted with a quadrature encoder, the output of which is read into the Labjack U6 data acquisition device, which streams data to a notebook computer via USB interface. An executable generated in MATLAB then saves the streamed data to a file while populating an on-screen display.

This MATLAB script also provides on-screen instructions to the subject and administrator, including printed text, audio and video content. During the paced tapping test, an audio file with metronome tapping cues is played. This audio file is 
split between headphones worn by the subject and an analog input on the Labjack, which records the audio synchronously with the position data from the quadrature encoder.

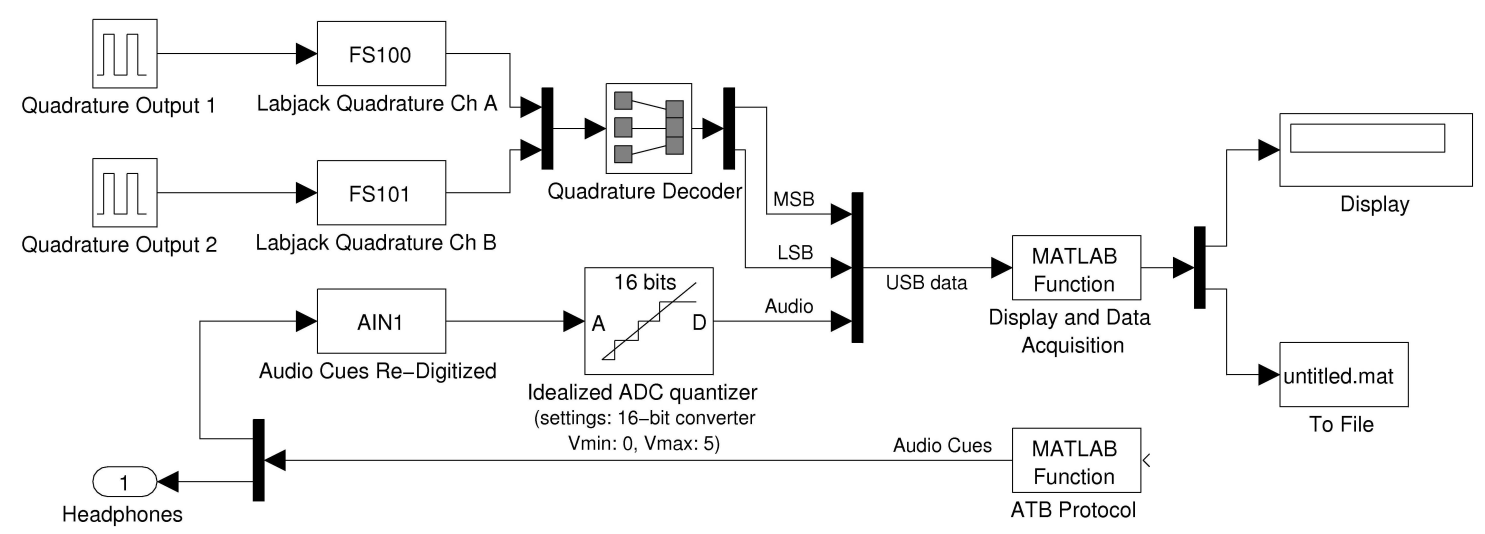

Figure 1: Signal diagram for complete system.

\subsection{Hardware}

Both devices are designed to measure the full range of motion required for a tapping task with minimal restriction of the subject's movement, while at the same time enforcing compliance to reduce variability. The finger tapping device is designed to allow tapping of the end of the index finger against the second knuckle of the thumb, a task that is considered to be more sensitive to early stage Parkinson's than the traditional fingertip to thumbtip tapping prescribed by the UPDRS. This finger-toknuckle tapping is referred to as as "OHSU" tapping, and finger-to-thumbtip tapping as "UPDRS" tapping. The single finger task closely imitates the UPDRS while 
eliminating strategies evident in some keyboard tasks.

The foot tapping device measures the angle of the foot as the toes are tapped, rotating on the heel. This involves considerably less movement than the UPDRS leg agility item. It is possible that the more distal location of the movement may prove to be more responsive.

As mentioned above, these tapping devices can provide effectively continuous feedback to the subject, if desired. This makes the devices more intuitive and user friendly by allowing the subject to see how their movements affect measurements, while at the same time providing simple and instant verification that the device is working properly. Furthermore, visual feedback allows for possible future studies to implement tracking tasks, if desired.

\subsubsection{Finger Tapper}

The finger tapping device consists of an aluminum baseplate with a steel shaft, or mast, centered at about three quarters of the length. The mast supports an aluminum crossbar, or boom, which in turn supports the two encoder enclosures, which hang down over the baseplate. Hanging from the bottom of the encoder enclosures are the hand grips, thumbrest and manipulandum. The height of the boom on the mast is adjustable by a quick release pressure clamp. The angles of the encoder enclosures on the boom are also adjustable, being held in place by pressure fit rubber washers. 
Figure 2 shows the assembled device, and Figures 3 and 8 show the device in use.

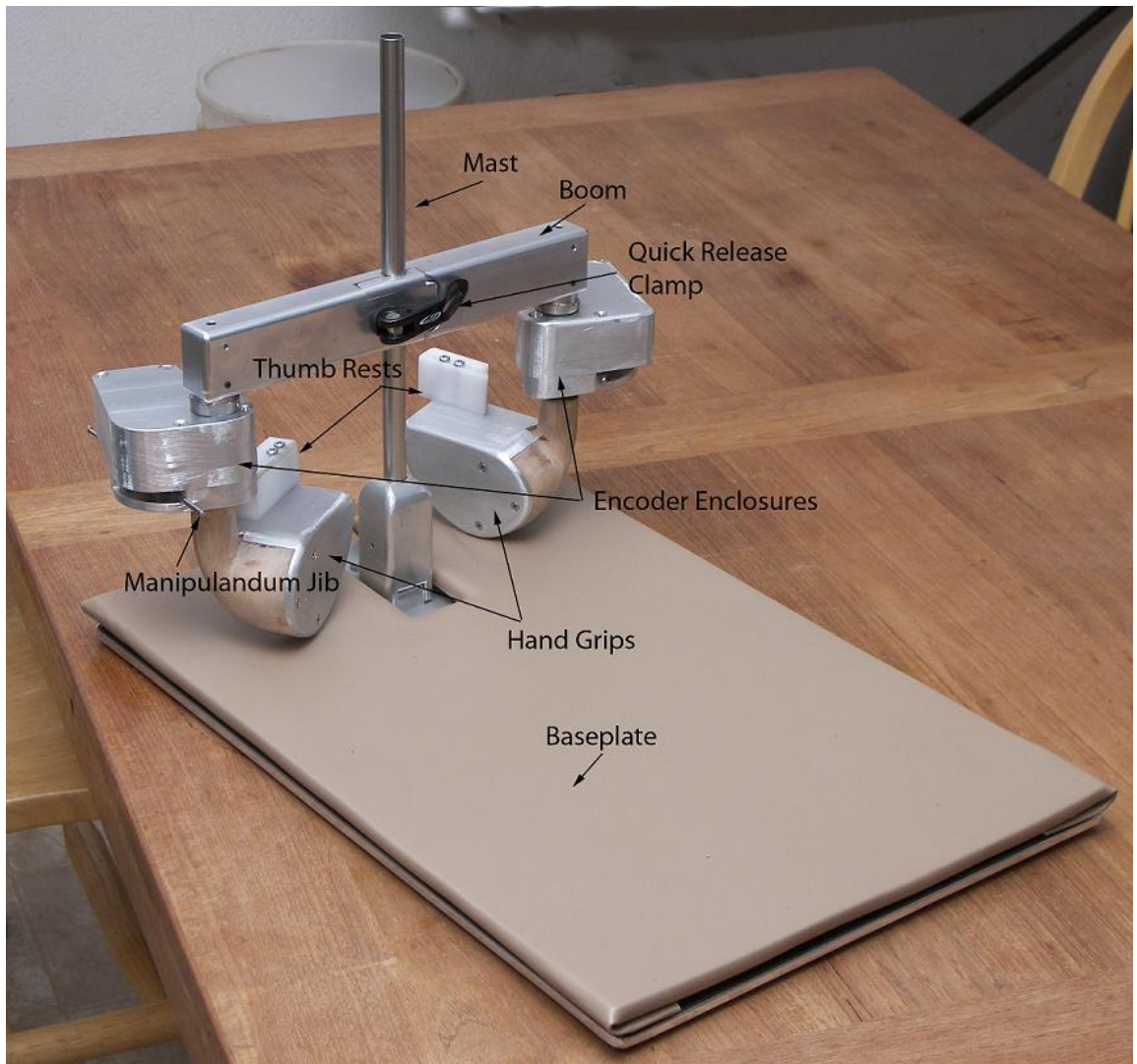

Figure 2: Finger tapping device with parts labeled.

The handgrip is made of wood and aluminum and features a guard to separate the other fingers from the index finger during tapping tests. The thumbrest is made of plastic and mounts on the top of the handgrip. The stationary position of the thumb enforces finger-to-thumb tapping, reducing variations due to test strategies. Figure 4 below is a detailed image of the hand grip and encoder assembly.

The mainupulandum consists of a narrow steel jib protruding from the encoder 


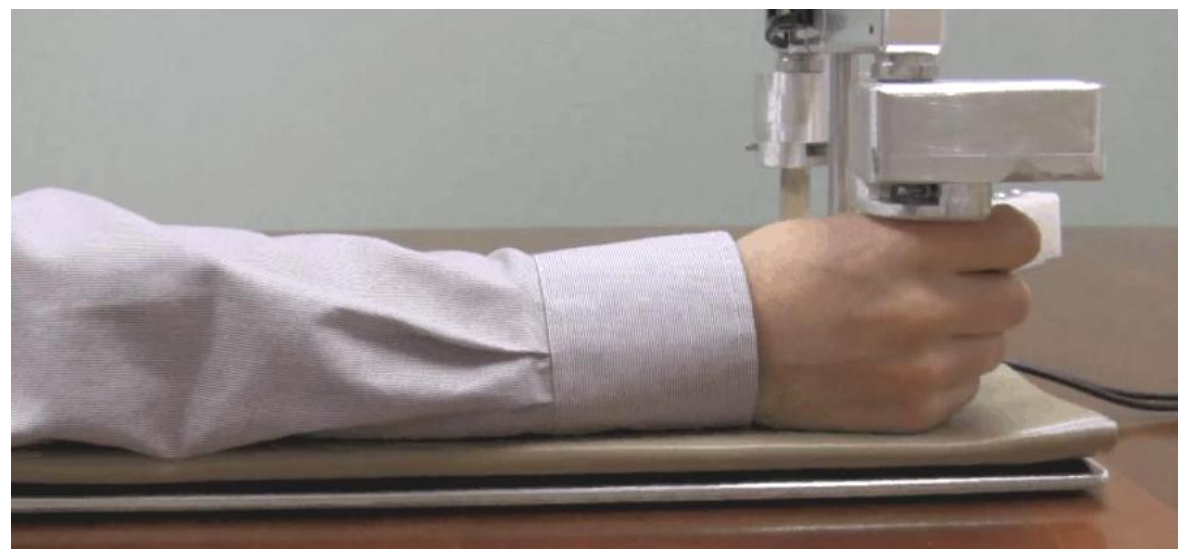

Figure 3: Finger tapping device showing subject arm position.

enclosure and ending in a small plastic bucket, or cap, for the finger tip. The cap has an aperture to accommodate large fingernails. The proximal end of the manipulandum jib connects to an axle supported by bearings on either side. The axle functions as the encoder shaft; the manipulandum jib forms a lever arm to rotate the encoder. A light spring returns the manipulandum to a starting position approximately 90 degrees from the thumbrest.

\subsubsection{Foot Tapper}

The foot tapping device consists of an aluminum base plate with a heel stop and two sets of brackets, each with an aluminum swing arm. The two swing arms are connected by a steel crossbar. An aperture is cut into the baseplate, and the crossbar is bent in such a way that it extends into the aperture when the crossbar is fully depressed. A light spring returns the crossbar and swing arms to their resting position 


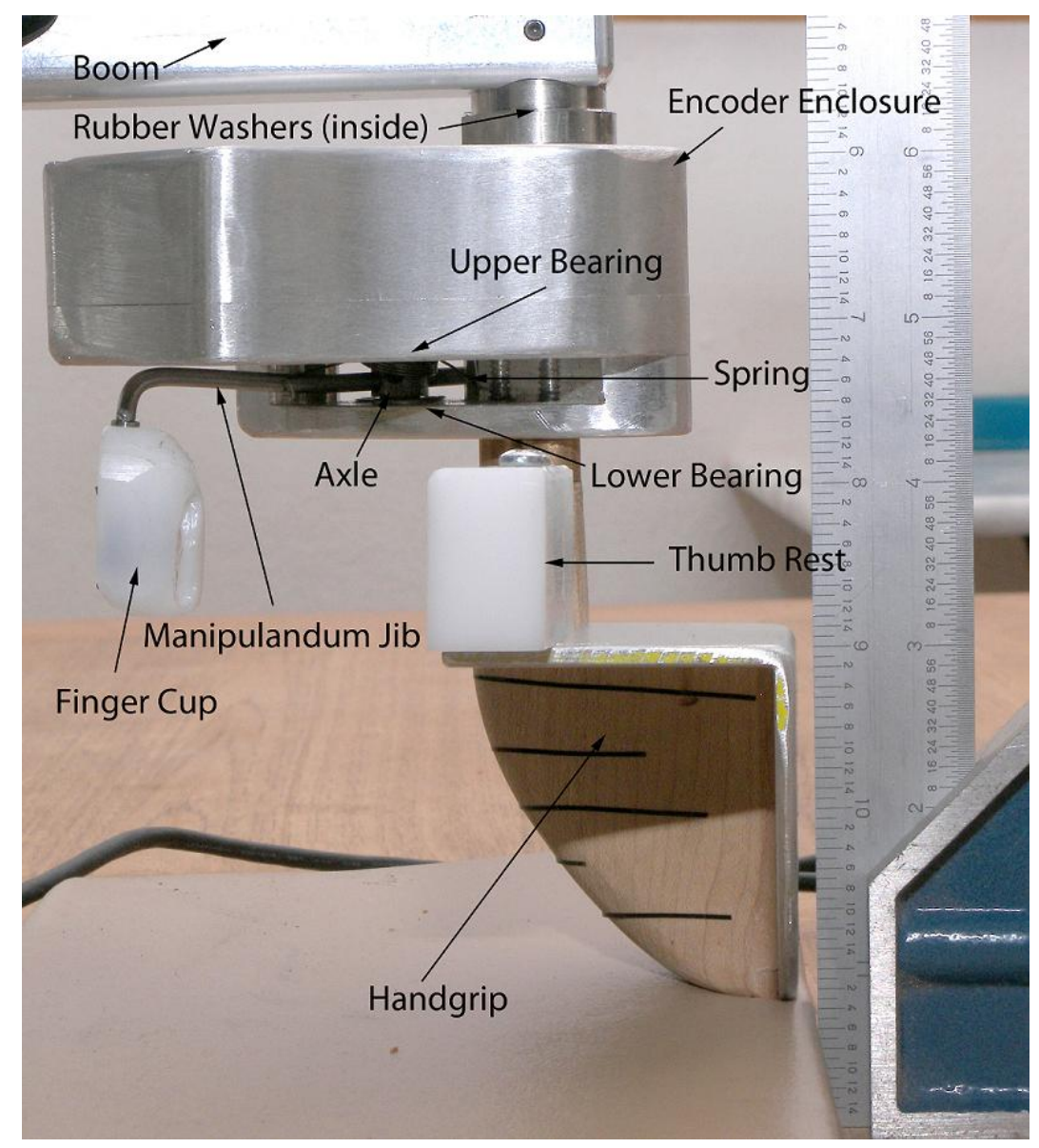

Figure 4: Detail of finger tapping device hand grip and encoder assembly.

at about 90 degrees off the baseplate. One of the swing arms is attached to a rotary encoder shaft mounted in a bearing just above the baseplate. The swing arm serves as a lever arm to rotate the encoder. Figure 6 below shows the assembled foot tapper with parts labeled.

The baseplate features screw down spikes to hold it in position on carpet. Grip tape and a curved heel rest reduce foot movement while tapping, and rubber pads 


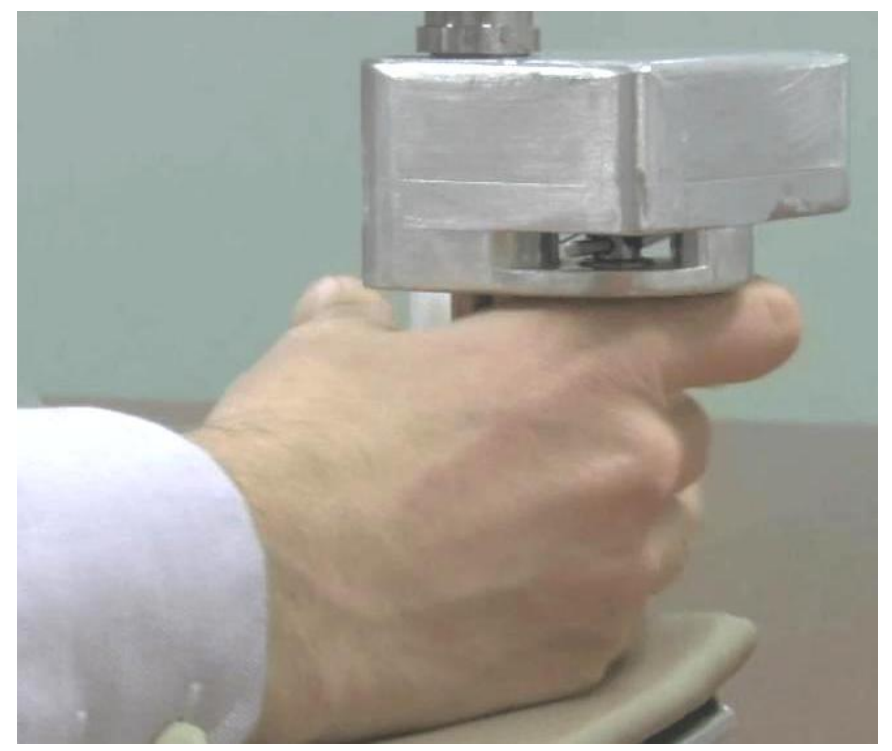

Figure 5: Finger tapping device handgrip showing subject hand position.

prevent the swing arms from hitting the base plate, reducing noise and subject feedback.

\subsubsection{Transducers}

The angular position transducers are identical for both devices. Each device is fitted with a HEDM-5600 J06 rotary incremental quadrature encoder (Avago Technologies, San Jose CA) with a resolution of 1024 steps per revolution, or about .35 degrees per step. There are no stops on the encoder, allowing unlimited full rotations and the absence of mechanical contact within the encoder reduces wear on the transducer. The encoder wheel is mounted on a shaft which is supported by a bearing system designed for the purpose of reducing the resistance to movement and required torque. 


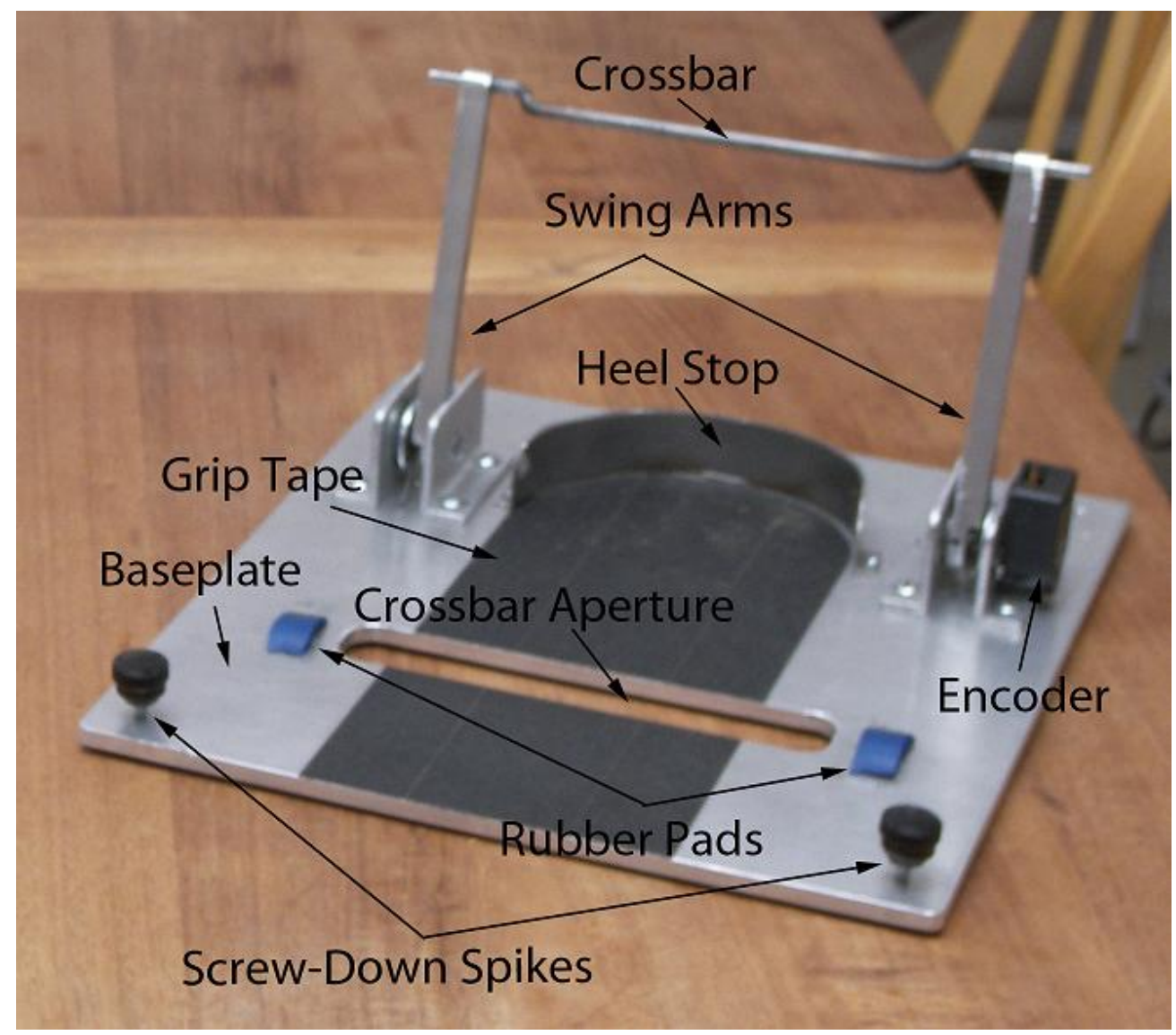

Figure 6: Foot tapping device with parts labeled.

The quadrature encoder provides position data with predictable quantization noise, predictable power draw and minimal EMI at the expense of additional signal processing. The quadrature must either be decoded with additional hardware or read as two digital streams and decoded in software. We have chosen a data acquisition module that decodes quadrature and passes it to the PC via USB. 


\subsubsection{Data Acquisition Module}

The encoder interfaces with the PC through an external data acquisition system, the LabJack U6 (LabJack Corporation, Lakewood CO), sampling at $10 \mathrm{kHz}$. This device has two on-board timers allowing it to perform quadrature decoding of the two pulse channels from the encoder. The LabJack is also used to capture the audio clicks used to cue the subject to tap at specific rates. Position and audio data is then sent to the PC as three 16 bit packets: LSB, Audio, MSB.

\subsubsection{Notebook Computer}

The PC used to capture data from the LabJack is an Acer Aspire 1 (Acer Inc., Taipei Hsien, Taiwan). This notebook PC has a small footprint, is inexpensive, and has built in wireless capability. The operating system is Windows XP.

The PC provides instructions to the administrator and subject, as well as cues for paced tapping, as described in the protocol below. A second monitor is provided for the subject display, including video instructions and visual feedback for practice

sessions. Audio cues are taken from the PC headphone jack and split between an analog input on the Labjack and the subject display and headphones. The audio cues are re-recorded by the data acquisition system to account for unknown latency and DAC rates in the Aspire's built in sound bus. 


\subsubsection{Headphones}

Headphones are provided to provide pacing cues to the subject while reducing feedback during the paced tapping test, both due to the noise of the device and unconscious cues from the administrator. The headphones have the additional advantage of restricting the metronome pacing to the subject, which reduces disturbance to the clinical environment.

\subsection{Data Acquisition Software \& Graphical User Interface}

The graphical user interface provides step-by-step instructions to the administrator to insure that the test is uniformly administered across subjects. The list of steps is referred to as the test protocol, described in Section 5.2. Each item of the protocol is represented by a radio button running down the side of the screen. Each button is described in detail below.

\subsubsection{Opening Screen}

Figure 7 is a screen capture from the opening screen.

The opening screen provides instructions to the administrator to record subject information. The specific information recorded and the entry methods are listed in Table 2 below. 


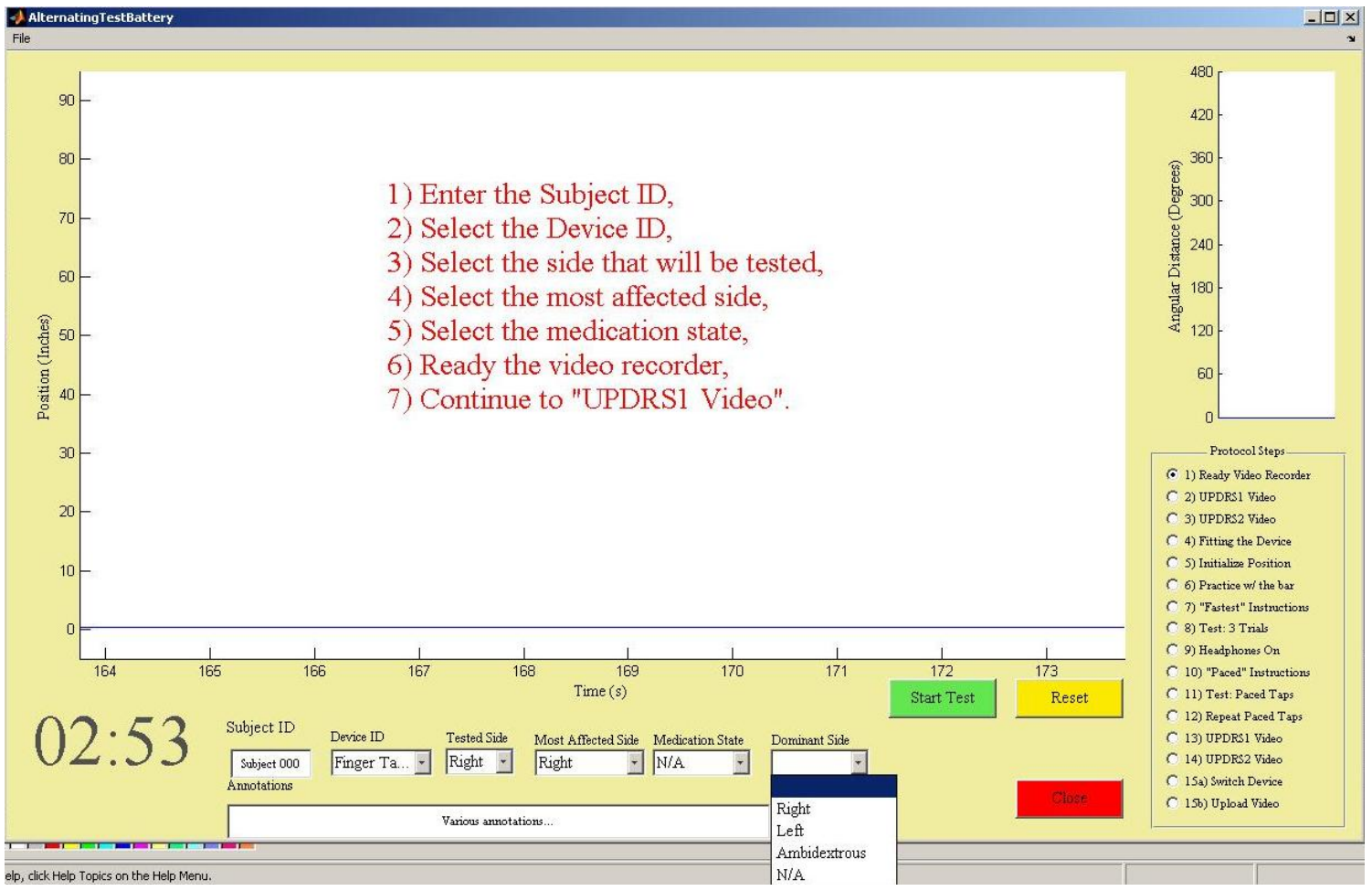

Figure 7: Data acquisition software opening screen

\begin{tabular}{lll} 
Information & Entry Method & Options \\
\hline \hline Enter the subject ID & Text Entry Box & (not applicable) \\
Select the device ID & Pull Down Box & 'Finger Tapper' or 'Foot Tapper' \\
Select the side that will be tested & Pull Down Box & 'right', 'left' or 'NA' \\
Select the most affected side & Pull Down Box & 'right', 'left' or 'NA' \\
Select the medication state & Pull Down Box & 'on', 'off' or 'NA' \\
Select the dominant side & Pull Down Box & 'right', 'left', 'Both' or 'NA' \\
\hline
\end{tabular}

Table 2: Subject information interface.

This screen also instructs the administrator to "Ready the video recorder" and then "Continue to UPDRS video". After entering the subject's information, the administrator selects the "UPDRS 1 Video" radio button, which opens the next screen. 


\subsubsection{UPDRS 1}

Selecting the "UPDRS 1 Video" radio button causes a video instruction to play in windows media player on the subject display. In the case of the finger tapper, the video instructs the subject to tap fingertip to thumbtip. In the case of the foot tapper, they are instructed to stomp their foot on the ground as high and as fast as possible. The administrator then records the activity with a video camera for later rating. After the video finishes, the subject display shows position display and the velocity bar, while the administrator display instructs them to continue after recording.

\subsubsection{UPDRS 2}

Selecting the "UPDRS 2 Video" radio button causes another video instruction to play in windows media player on the subject display. In this case, if the current device is the finger tapper, the subject is instructed to tap the fingertip against the first knuckle of the finger. If the current device is the foot tapper, the subject is instructed to tap their toes on the ground as fast and as big as possible. The administrator then records the task with a video camera for later rating. With the exception of slightly different tasks in the instructional videos, this step is the same as the previous one. After the video finishes, the subject display shows position display and velocity bar, while the administrator display instructs them to continue after recording. 


\subsubsection{Fitting the Device}

Selecting the "Fitting the Device" radio button causes a video instruction on the physical use of the device, either finger tapper or foot tapper, to play in windows media player on the subject display. Figure 8 below shows a capture from the finger tapper instructional video.

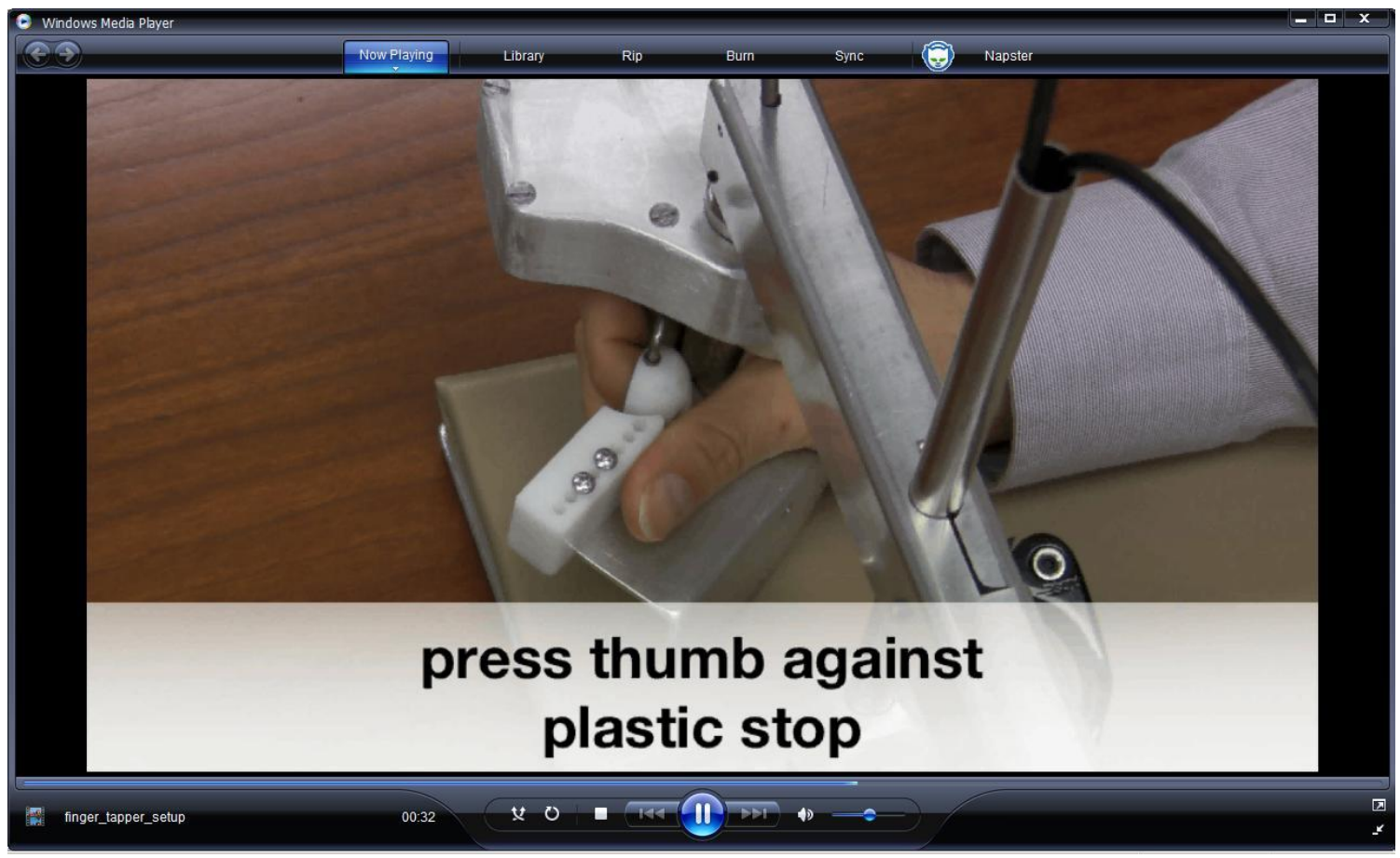

Figure 8: Capture from Finger Tapper instructional video shown during "Fitting the Device".

While the video is playing, the subject learns to operate the device, assisted the administrator if necessary. The administrator display instructs the administrator to continue to the next step after the subject is comfortable and has proper command of the device. After the video is completed, the subject display returns to the position 
display and velocity bar.

\subsubsection{Initialize Position}

Selecting the "Initialize Position" radio button brings up instructions on the administrator display to click the reset button after having the subject fully depress the manipulandum. While "Initialize Position" is selected, the reset button causes the current position of the manipulandum to be set to zero and all future positions to be measured with respect to that point. If the radio button is not selected, the reset button does not have this effect. The administrator display instructs them to select "Practice with Bar" when they are ready to continue to the next step.

\subsubsection{Practice with the Bar}

Selecting the "Practice $\mathrm{w} /$ Bar" radio button causes a video instruction specific to the foot or finger tapper to play on the subject display. This video instructs them to tap as large and as fast as possible to raise the velocity bar. After the video, the subject display shows the velocity bar with instructions to try to make it go as high as possible. The administrator display instructs them to continue when the subject has finished practicing. Figure 9 below shows a capture from the foot tapper practice instructional video. 


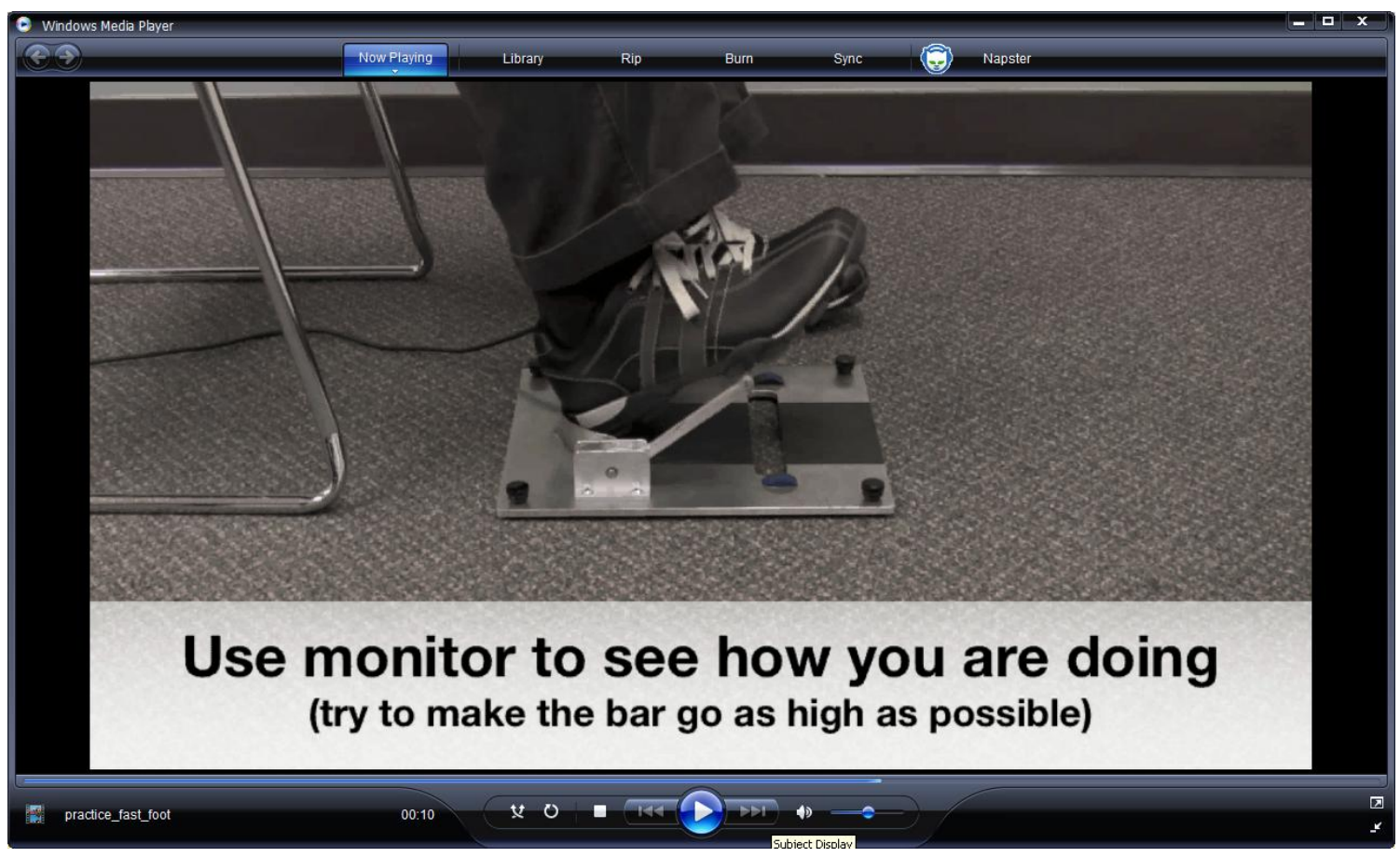

Figure 9: Capture from foot tapper practice video shown during "Practice With the Bar".

\subsection{7 "Fastest" Instructions}

Selecting the "Practice with the Bar" radio button causes an audio instruction on the fast tapping test to play. The Subject display shows the velocity bar and "Fastest" instructions. The administrator display instructs them to select "Test: 3 Trials" and press the start button when the subject is ready to continue.

\subsubsection{Trials Fast Test}

The three trials fast test consists of three ten second trials during which the subject is asked to tap as high and as fast as possible. Between each trial the subject is 
shown their score in taps per minute and allowed to rest for ten seconds. The angular position data for each trial is stored as a ".mat" file. Table 3 below summarizes the audio and text cues displayed to the subject during the test.

\begin{tabular}{|c|c|c|}
\hline Time & Audio Cue & Subject Display \\
\hline$\overline{0}$ & "start" & (Velocity Bar) \\
\hline \multirow[t]{3}{*}{10} & "Take a rest before & "Rest" (10 Second rest timer) \\
\hline & the next round" & "Your score is $\mathrm{N}$ taps per minute \\
\hline & & for the 1rst trial.Try and do better!" \\
\hline 20 & "three... two... one... Start!" & (Velocity Bar) \\
\hline \multirow[t]{2}{*}{30} & "Take a rest before the & "Rest" (10 Second rest timer) \\
\hline & & $\begin{array}{l}\text { "Your score is } \mathrm{N} \text { taps per minute } \\
\text { for the } 2 \text { nd trial. Try and do better!" }\end{array}$ \\
\hline 40 & "three... two... one... Start!" & (Velocity Bar) \\
\hline 50 & "You have completed the test." & $\begin{array}{l}\text { "Your score is } \mathrm{N} \text { taps per } \\
\text { minute for the 3rd trial." } \\
\text { "You have completed the test." }\end{array}$ \\
\hline
\end{tabular}

Table 3: Subject Cues for 3 Trials Fast Test.

Selecting the "Test: 3 Trials" radio button causes the subject display to show only the velocity bar, while the administrator display instructs them to press the start button to begin the test. When the start button is pressed, data recording begins, a ten second timer appears on the administrator screen, and an audio file plays. The audio file cues the subject to begin tapping, and then to rest after ten seconds. At this time a 10 second "rest" timer appears on the subject screen, and the subject is shown their score as "Your score is $\mathrm{N}$ taps per minute for the first trial. Try and 
do better!". At the end of the ten second rest period, an audio cue counts down as "three... two... one... Start!" and another ten second trial begins. This repeats until the subject has completed three trials, after which the subject display shows the additional message "You have completed the test." The administrator display shows scores from all trials during the entire test. At this time the administrator display instructs them to "Select Headphones on" when they are ready to continue.

\subsubsection{Headphones On}

Selecting the "Headphones On" radio button causes a video instructing the subject to put on the headphones to play on the subject display. The administrator display instructs them to assist with headphones and proceed to paced instructions.

\subsubsection{Paced Instructions}

Selecting the "Paced Instructions" radio button causes a video instruction for the paced tapping test to play on the subject display. The video instructs the subject to tap along to the recorded beat and to continue tapping after the beat fades, as

described in section 4.2.11, below. The administrator display instructs them to select "Test: Paced Taps" and press the start button when they are ready to begin. The data from this test is not considered in this thesis. 


\subsubsection{Paced Tapping Test}

The paced tapping test consists of six consecutive trials, each separated by a ten second rest. Each trial consists of a paced portion, where a metronome pacing cue is provided to the headphones, and an un-paced portion, where pacing cue stops and the subject attempts to continue to tap at the previously provided pace. With each trial, the rate of tapping is increased; the tapping frequencies are $1.5 \mathrm{~Hz}$ (taps per second), $2 \mathrm{~Hz}, 2.5 \mathrm{~Hz} 3 \mathrm{~Hz}, 3.5 \mathrm{~Hz}$ and $4 \mathrm{~Hz}$. This test is taken with the head phones on and the eyes closed to reduce the feedback to the subject, as they are to tap based only on internal control if possible. Playing the audio cues in headphones reduces the possibility of involuntary, sympathetic tapping by the administrator, as well as reducing disturbances to the clinical environment. As mentioned in 4.2.10, the data from this test is not considered in this thesis.

\subsubsection{Repeat Paced Tapping Test}

Selecting the "Repeat Paced Taps" radio button repeats the paced tapping test exactly as previously described. At the end of the test, a video instructing the subject to remove their headphones plays on the subject display. The paced tapping test was not repeated for the subjects whose data is analyzed in this thesis due to time constraints, but may be repeated in future studies. 


\subsubsection{Repeat UPDRS $1 \& 2$}

Selecting the "UPDRS 1 Video" and "UPDRS 2 Video" radio buttons have exactly the same response as above in sections 5.3.2 and 5.3.3. The purpose is to cause offline raters to rate the same subject in the same state twice, so as to establish intra-rater reliability. The tapping video was not repeated for the subjects whose data is analyzed in this thesis due to time constraints, but may be repeated in future studies.

\subsubsection{Switch Devices}

Selecting the "Switch Devices" radio button causes the subject display to show a blank screen. The administrator display instructs them to select the other device ID and repeat the protocol steps. The administrator must physically unplug the current device (either finger or foot tapper) and connect the other one. They then return to the UPDRS1 Video button and repeat the protocol from there with the other device.

\subsubsection{Upload Video}

Selecting the "Upload Video" radio button causes Windows explorer to open the ATB Data directory. The administrator then connects the video camera used for UPDRS recordings and drags all of the avi files into this directory. This is typically the last step before clicking on the "close" button. 


\subsubsection{The "Close" Button}

When the "Close" button is pressed, all data and video files are zipped and saved. If the software can find an internet connection, the data is uploaded to BSP lab data servers for offline processing. The data is also saved locally on the PC used to administer the test.

\subsection{Data Analysis Software}

The data analysis can be divided into three discrete tasks: Populating the Data Structure, Data Analysis, and Statistical Comparison. In general terms, the Populating the Data Structure step assembles position data from multiple channels within a trial and then assembles and data structure from multiple trials. The Data Analysis step computes metrics for each trial within the data structure, and the Statistical Comparison step analyses metrics and test conditions for correlations. Each step is considered separately in the sections below.

\subsubsection{Populating the Data Structure}

The first step in analysis is to combine the .mat files from all of the subject's trials in to a single data structure for analysis as conceptualized in Figure 10 below. As this is a fairly computationally intense process, the resulting data file is saved and reloaded for later analysis, and updated only when new subject data is available. 


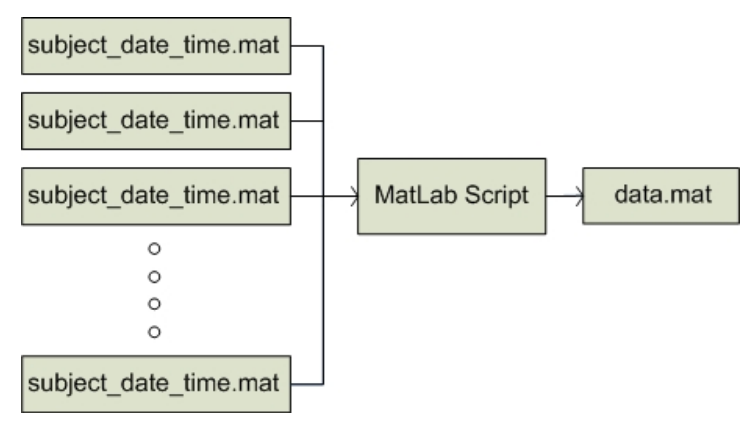

Figure 10: Block diagram of data structure population.

Each subject takes the 3 Trials Fast test once with each device in the off medication state, then repeats it in the on state. Each trial produces a .mat file, resulting in 12 files per subject all together. Each .mat file is a data structure with 13 fields, detailed in Table 4.

\begin{tabular}{ll} 
Field & Description \\
\hline \hline annotations & String assigned in the Protocol GUI by the test administrator \\
subjectID & Subject's identity number \\
data & Data recorded by Labjack, stored as one vector interleaved \\
testDate & Number representing the time and date that data was saved \\
sideTested & String assigned in protocol - 'right', 'left' or 'NA' \\
mostAffectedSide & String assigned in protocol - 'right', 'left' or 'NA' \\
medicationState & String assigned in protocol - 'on', 'off' or 'NA' \\
dominantSide & String assigned in protocol - 'right', 'left', 'Both' or 'NA' \\
testTag & String assigned by protocol software: \\
& '3 Trials: Fast w PTFB' or 'Paced Tapping' \\
nTrial & Number of trial in 3 trials test \\
testDuration & Duration of data recording in seconds \\
scanRate & Sample rate per channel, set to 10 kHz \\
num_channels & Number of channels interleaved in stream \\
\hline
\end{tabular}

Table 4: Data Fields in Unprocessed .mat Files.

A MATLAB script is written which loads each of the .mat files from a target directory structure and creates a data structure with the fields in Table 5. With 
the exception of the angle field, the value in each field is directly assigned from a field in the raw structure. The angle field consists of a vector constructed from the data field in the raw structure, which in turn consists of three channels interleaved. The first sample represents the least significant bits of the position decoded from the quadrature streams by the LabJack, the second is a sample of the audio track from the analog in channel of the Labjack, the third is the most significant bits of the decoded position. The MATLAB script assembles the least and most significant values. The result is then decimated to a sample rate of $1000 \mathrm{~Hz}$ and written to the angle field in the data structure.

\begin{tabular}{ll} 
Field & Description \\
\hline \hline sampleRate & Sample rate after down-sampling, set to 1000 Hz \\
angle & Vector of position data \\
frequencies & Vector of nominal cue frequencies for paced tapping \\
tPrompts & Vector cue times for paced tapping \\
deviceName & String assigned in protocol - 'fingerTapper' or 'footTapper' \\
iSubject & Subject's identity number \\
annotations & String assigned in the Protocol GUI by the test administrator \\
sideMostAffected & String assigned in protocol - 'right', 'left' or 'NA' \\
dateNumber & Number representing the time and date that data was saved \\
motorState & String assigned in protocol - 'on', 'off' or 'NA' \\
cTrial & Number of trial in 3 trials test \\
duration & Duration of data recording in seconds \\
testSummary & String assigned by protocol software - ' \\
& '3 Trials: Fast w PTFB' or 'Paced Tapping \\
sampleRateFactor & Sample Rate Factor \\
\hline
\end{tabular}

Table 5: Data Structure Fields Assigned Directly. 


\subsubsection{Data Processing}

After a data structure is assembled from all the trials, another MATLAB script processes the three trials data to obtain metrics for comparison with test conditions. Although Paced Tapping data is present in the data structure, this thesis considers only the three trials test. The Data Processing step can be further subdivided into two tasks- sorting and event detection.

\section{Sorting}

If the data structure exists, it is loaded, otherwise it must be populated as above. A four column matrix is constructed based on the motorState and testSummary fields, where the data structure indexes of 'on' '3 Trials: Fast $w$ PTFB' trials are placed in the first column, 'off' '3 Trials: Fast w PTFB' in the second. 'On' and 'Paced Tapping' trial numbers are placed in the third column, 'Off' and 'Paced Tapping' in the fourth. The resulting matrix is referred to as the "Address Matrix". The analysis in this thesis will be on the first two columns of the address matrix, '3 Trials: Fast w PTFB' in the on and off motor states.

\section{Event Detection}

The concept of an "event" is defined in the most general sense as any set of samples comprising a region of interest. To find events, a median filter is applied 
angular position data to flatten peaks and troughs, and regions with greater than 10 consecutive identical samples are defined as "events". The events are then classified as described in Table 6. The numbers of the starting and ending samples of all of the events in a given trial are collected as a vector and appended to the trial's entry in the data structure. Each event in this vector in turn has a substructure which includes fields such as amplitude, duration, and classification. The complete set of fields are tabulated in Table 7 .

\begin{tabular}{ll} 
Event & Description \\
\hline \hline Peak & $\begin{array}{l}\text { An event with an amplitude higher than it's } \\
\text { previous and subsequent neighbors }\end{array}$ \\
\hline Trough & $\begin{array}{l}\text { An event with an amplitude lower than it's } \\
\text { previous and subsequent neighbors }\end{array}$ \\
\hline Left Saddle & $\begin{array}{l}\text { An event with an amplitude higher than it's } \\
\text { previous and lower than it's subsequent neighbors }\end{array}$ \\
\hline Right Saddle & $\begin{array}{l}\text { An event with an amplitude lower than it's } \\
\text { previous and higher than it's subsequent neighbors }\end{array}$ \\
\hline High Trough & $\begin{array}{l}\text { A subset of troughs with an amplitude within one } \\
\text { standard deviation of the mean peak amplitude }\end{array}$ \\
\hline True Trough & $\begin{array}{l}\text { A subset of troughs with an amplitude within two } \\
\text { standard deviations of the mean trough amplitude }\end{array}$ \\
\hline Indeterminate & An event which does not meet any of the previous criteria \\
\hline
\end{tabular}

Table 6: Descriptions of event classifications.

Events are classified as peaks, troughs, right saddles or left saddles based on their amplitudes in degrees with respect to their nearest neighbors. Figures 11 and 12 are plots of angular position with detected events highlighted. Red events are peaks, green are troughs, blue are left saddles, olive are right saddles, and purple are 
indeterminate.
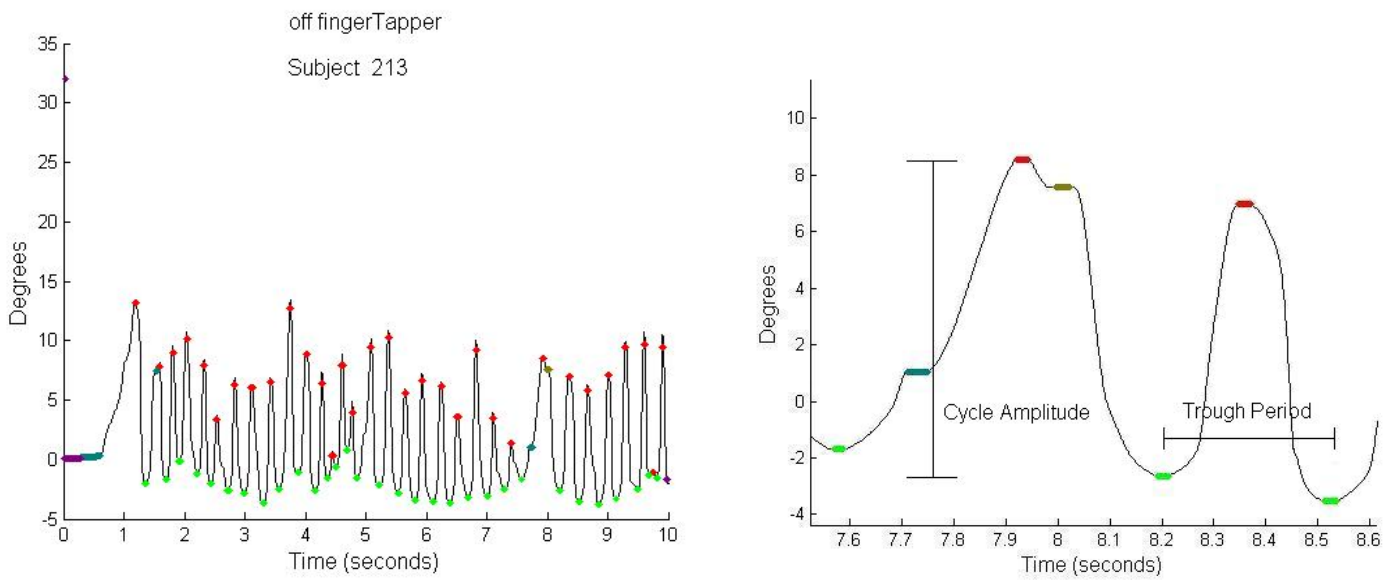

Figure 12: Detail from the same trial showing Figure 11: Plot of a subject trial with detected trough, left saddle, peak and right saddle. events highlighted.

The duration of each tap is computed as the period between each "true" trough, where true troughs are those below a threshold angle defined as two standard deviations of the mean trough amplitude, as seen in Figure 12. The number of taps is computed as the number of true troughs detected. Cycle amplitude is computed for each peak as the difference between peak and adjacent trough angles, again illustrated in Figure 12. Mean and standard deviations of amplitudes and tap durations are computed and appended to each trial's entry in the main data structure, as are the number of taps and duration of each event classification in samples. Table 8 below shows the complete set of computed fields. These fields will be used to compute the various metrics in Section 5.3.1. 


\begin{tabular}{ll} 
Field & Description \\
\hline \hline startTime & Starting time of event, in samples \\
amplitude & Angle of event, in degrees \\
eventNumber & Chronological number of event \\
endTime & Ending time of event, in samples \\
length & Elapsed time of event, in samples \\
previousAmp & Angle of previous event, in degrees \\
previousLength & Elapsed time of previous event, in samples \\
previousEnd & Ending time of previous event, in samples \\
nextAmp & Angle of next event, in degrees \\
nextLength & Elapsed time of next event, in samples \\
nextEnd & Ending time of next event, in samples \\
peak & Boolean 1 if event is a peak, 0 otherwise \\
trough & Boolean 1 if event is a trough, 0 otherwise \\
rightSaddle & Boolean 1 if event is a right saddle, 0 otherwise \\
leftSaddle & Boolean 1 if event is a left saddle, 0 otherwise \\
troughPeriod & Duration between this event, if a trough, \\
& and the next trough, in samples \\
eventTime & Sample at which event is considered to have occurred \\
\hline
\end{tabular}

Table 7: "eventStructures" Substructure Fields.

\begin{tabular}{ll} 
Field & Description \\
\hline \hline events & Vector of starting and ending times of \\
& detected events \\
meanTroughPeriod & Mean of durations between troughs \\
stdTroughPeriod & Standard Deviation of durations between troughs \\
troughPeriods & Vector of durations between troughs \\
numberOfTaps & Number of thresholded troughs \\
eventStructures & Substructure of detected events \\
cycleAmplitudes & Vector of cycle amplitudes \\
meanCycleAmplitudes & Mean of cycle amplitudes \\
stdCycleAmplitudes & Standard deviation of cycle amplitudes \\
totalPeakTime & Total duration of "peak" events, in samples \\
totalTroughTime & Total duration of Trough" events, in samples \\
totalRightSaddleTime & Total duration of "RightSaddle" events \\
totalLeftSaddleTime & Total duration of "LeftSaddle" events \\
totalIndeterminateEvntTime & Total duration of events not otherwise classified \\
totalHighTroughTime & Total duration of "HighTrough" events \\
\hline
\end{tabular}

Table 8: Data Structure Fields Assigned to Trials in Data Processing. 


\section{Methodology}

\section{$5.1 \quad$ Subjects}

Subjects were recruited from the Movement Disorders Clinic at the Oregon Health Sciences University (OHSU) neurology department. Subjects were between the ages of 50 and 80 and have confirmed diagnosis of Parkinson's disease, as well as demonstrated responsiveness to levodopa. For inclusion in the study, subjects had to be able to stand and walk unassisted and follow verbal instructions. Subjects with medical or psychiatric conditions that prevent safe testing or operation of the devices were excluded, as were subjects with medical conditions other than Parkinson's disease which result in significant impairment of movement.

When tested, subjects arrived at the test site in the morning after withholding their medication for at least 12 hours. The off medication state was verified by a clinician and a UPDRS rating was performed. The subjects performed the the protocol as described in Table 9, along with some additional tests for related studies. Subjects performed the test on their most affected side. After completing the tests, they took an extra large dose (about $120 \%$ of their usual dose) of levodopa and waited one hour. The subjects were then re-examined by the clinician to verify an on medication state, and a new UPDRS was performed. The subjects then repeated the test batteries. 


\subsubsection{Motor UPDRS}

The metrics discussed in Section 5.3.1 are compared for correlation to the motor subsection of the UPDRS, which consists of 27 items directly related to movement. These items include assessments of rigidity, tremor, slowness of movement, gait and facial expression. Assessments were provided by the clinical research assistants who administered the protocol, and are not blinded.

\subsection{Protocol}

The protocol is delineated by the data acquisition software, as described in Section 4.2, and provides step by step instructions to the administrator and subject. The protocol steps are summarized in Table 9 .

\begin{tabular}{lll} 
& Protocol Item & Purpose \\
\hline \hline 1 & Subject information is recorded & Provides the test conditions \\
2 & Video of UPDRS tapping (finger tapper) & Tapping is recorded for verification \\
& Video of leg agility (foot tapper) & $\cdot$ \\
3 & Video of OHSU tapping (finger tapper) & $\cdot$ \\
& Video of toe tapping (foot tapper) & Insures the basic operation of the device \\
4 & Fitting the device & Begin tap at 0 degrees for ease of analysis \\
5 & Initialize position & Helps to reduce the learning effect \\
6 & Practice for three trials fast test & Tests amplitude, maximum rate of \\
7 & Three trials fast test & tapping and hesitations and halts \\
& & Tests the effect of the loss of pacing tones \\
8 & Paced tapping Test & Each item is done with the finger \\
9 & Repeat for other device & tapper and foot tapper \\
& &
\end{tabular}

Table 9: Protocol steps for each device. 
Each of these items is intended to be executed in order, although items may be restarted or repeated at the discretion of the administrator. The interface provides an annotation field in which any irregularities can be noted. This section describes the intentions and relevance of the protocol items, while the actual execution of each is described in Section 4.2 .

\subsubsection{Subject Information}

The subject's tested side, most affected side, dominant side, and medication state are recorded. These will be used to establish the conditions for the data set. The subject ID and device ID (i.e Finger Tapper or Foot Tapper) were also assigned at this point, as certain parameters in the data acquisition software are different for each device.

\subsubsection{Video Recording}

A video recording of the tapping task was made to serve as a check on subject's performance, and to confirm the operation of the devices. The field of view of all video recordings was limited to hands or feet to prevent subject identification.

\subsubsection{Three Trials Fast Test}

The Three Trials Fast test consists of three ten second trials during which the subject is asked to tap as large and as fast as possible. Between each trial the subject is 
allowed to rest for ten seconds. The data collected is angular position data, and metrics are computed as described in Section 4.3.2. The repetition of three simple tasks allows us to estimate repeatability and helps to suppress the learning effect.

\subsubsection{Paced Tapping Test}

The paced tapping test consists of six consecutive paced trials, each separated by a ten-second rest, as described in Section 4.2. The data recorded is angular position, as well as raw audio recordings of the pacing cues. The audio is re-recorded synchronously with the position data to avoid unknown or ambiguous latencies in the playback and sampling, particularly as the software is compiled to a standalone executable from MATLAB. The data from this test is not considered in this thesis.

\subsection{Metrics}

For the purpose of this section, the word metric refers to a quantity which is compared to a condition to assess symptoms. The word condition refers to the combined medication state and device. All of the metrics described are derived from the measured quantity of angular position, and each is associated with a condition. For convenience, metrics are subdivided into two broad categories: amplitude and period metrics and event duration metrics. Each of these is described in detail in the relevant section below. 


\subsubsection{Amplitude and Period Metrics}

The amplitude and period metrics consist of the mean trough period, standard deviation of trough period, mean cycle amplitude, standard deviation of cycle amplitude, and the number of taps. These are listed in Table 10. Each of these quantities was computed and appended to the data structure in Section 4.3.2. The mean trough period represents the average period between valid troughs for one trial of the three trials fast task, and the standard deviation is the standard deviation of the same set of periods. Cycle amplitude is computed as the difference in angular position value between each peak and adjacent trough, the mean and standard deviation of the set of amplitudes within a trial is computed in the same manner as above. The number of taps represents the number of times the subject completes full tapping motion, and is computed by counting the number of valid troughs detected.

\begin{tabular}{l} 
Metric \\
\hline \hline mean of trough period \\
standard deviation of trough period \\
mean of cycle amplitude \\
standard deviation of cycle amplitude \\
number of taps \\
\hline
\end{tabular}

Table 10: List of amplitude and period metrics.

\subsubsection{Event Duration Metrics}

The event duration metrics consist of the total peak time, total trough time, total right saddle time, total high trough time, and total indeterminate event time. These 
are tabulated in Table 11. Each metric is reported in samples. The total time elapsed for each type of event was computed and appended to the data structure in Section

\begin{tabular}{l} 
Metric \\
\hline \hline total peak time \\
total trough time \\
total right saddle time \\
total high trough time \\
total indeterminate event time \\
\hline
\end{tabular}

Table 11: List of event duration metrics.

\subsection{Conditions}

For each metric, the average of all three trials for each condition was computed for each subject. This resulted in a score (an average of means or average of standard deviations) for each subject, which was then plotted versus the motor UPDRS scores assigned by the clinician. Correlation coefficients were then computed between the two. This was repeated for each condition in Table 12.

\begin{tabular}{ll} 
Abscissa & Ordinate \\
\hline \hline motor UPDRS & Finger Tapper On Score \\
motor UPDRS & Finger Tapper On Score \\
motor UPDRS & Foot Tapper Off Score \\
motor UPDRS & Foot Tapper Off Score \\
\hline
\end{tabular}

Table 12: Conditions plotted for each metric. 


\subsection{Correlation Coefficients}

The amplitude, period and event duration metrics are compared to UPDRS rating scores using the built in MATLAB function corrcoef, which produces a matrix of correlation coefficients. The correlation coefficient is an indicator of the amount of variation in one variable that can be explained by variation in another. It varies from -1 to 1 , with -1 and 1 indicating a strong linear relationship and zero indicating no relationship. A general interpretation is that coefficients with an absolute value below .3 indicate a weak relationship, and coefficients above .7 indicate a strong relationship, assuming there is sufficient data to estimate the correlation.

\subsection{Effect Size}

To examine the relation to medication state, the effect size is computed. For each metric, the mean and standard deviation of the value of the metric for three trials is computed for both the on and off medication states. The effect size $d$ is then computed as:

$$
d=\frac{\mu_{\mathrm{off}}-\mu_{\mathrm{on}}}{\frac{1}{2}\left(\sigma_{\mathrm{off}}+\sigma_{\mathrm{on}}\right)}
$$

Where $\mu_{\mathrm{off}}$ is the mean of off medication trials, $\mu_{\mathrm{on}}$ is the mean of on medication trials, and $\sigma_{\text {off }}$ and $\sigma_{\text {on }}$ are the standard deviations of off and on medication trials, respectively. 
Effect size can be interpreted as a measure of how well the instruments can distinguish between the on and off medication states. If the two conditions both have low variance and widely separated means, a large effect size is obtained. As the variance of either condition increases, it becomes more difficult to distinguish between conditions, and effect size decreases. Practically speaking, most effect sizes are less than one, although there is no theoretical limit on the value. The effect size as computed for this thesis is similar to Cohen's standardized mean difference with pooled deviation. Cohen's rule of thumb for interpretation is that effect sizes below .2 are "small" effects, those over .8 "large" effects, and those in between are "medium", with the caveat that different disciplines and different data types might have different thresholds for description [41].

Effect size and difference of means are reported in Table 15 for amplitude and period metrics and Table 17 for event duration metrics. 


\section{$6 \quad$ Results \& Discussion}

\subsection{Data}

\subsubsection{Group Statistics}

Subject data were provided to the BSP laboratory identified only by subject number. Subject data sets were excluded at the discretion of the lab if instructions were not followed satisfactorily, devices failed or data was corrupted. Twenty two subjects are

included in the analysis. No age or gender information was provided. Handedness is established by the clinician by inquiry. Subject responses are tabulated in Table 13 , below.

\begin{tabular}{llll} 
Information & Right & Left & Both \\
\hline \hline Dominant Side & 20 & 1 & 1 \\
Most affected side & 16 & 6 & 0 \\
Tested side & 14 & 8 & 0 \\
\hline
\end{tabular}

Table 13: Number of subject responses.

Five subjects reported being right hand dominant and left side most affected, these subjects were tested on the left side. The single left handed subject reported the left side to be most affected.

The data analysis described produced 11 metrics in four conditions, producing 44 plots. The effect size for each metric for the finger and foot tapper produced an additional 22. As it is not immediately clear which if any are useful for assessment, coefficients are generated automatically for inspection, and tabulated in Tables 14, 
15, 16 and 17. Metrics with correlation coefficients greater than .3 are examined in Chapter 6.2.

\subsubsection{Amplitude and Period Metric Data}

The four conditions in Table 12 are computed for each of the five metrics in Table

10. Correlation coefficients with respect to the motor UPDRS are reported in Table

14, and effect sizes in Table 15. Select plots are presented in Chapter 6.2.

\begin{tabular}{llcrr} 
Metric & Device & Med State & p value & Correlation \\
\hline \hline Mean trough period & Finger & on & 0.607 & 0.113 \\
Mean trough period & Foot & on & 0.547 & -0.132 \\
Mean trough period & Finger & off & 0.979 & 0.006 \\
Mean trough period & Foot & off & 0.108 & -0.344 \\
St.Dev. trough period & Finger & on & 0.459 & 0.163 \\
St.Dev. trough period & Foot & on & 0.534 & 0.137 \\
St.Dev. trough period & Finger & off & 0.111 & 0.341 \\
St.Dev. trough period & Foot & off & 0.248 & -0.251 \\
Mean cycle amplitude & Finger & on & 0.777 & -0.062 \\
Mean cycle amplitude & Foot & on & 0.456 & -0.164 \\
Mean cycle amplitude & Finger & off & 0.152 & -0.309 \\
Mean cycle amplitude & Foot & off & 0.121 & -0.333 \\
St.Dev. cycle amplitude & Finger & on & 0.312 & 0.220 \\
St.Dev. cycle amplitude & Foot & on & 0.217 & 0.268 \\
St.Dev. cycle amplitude & Finger & off & 0.128 & 0.327 \\
St.Dev. cycle amplitude & Foot & off & 0.212 & 0.271 \\
Number of taps & Finger & on & 0.591 & -0.118 \\
Number of taps & Foot & on & 0.509 & 0.145 \\
Number of taps & Finger & off & 0.537 & 0.136 \\
Number of taps & Foot & off & 0.178 & 0.291 \\
\hline
\end{tabular}

Table 14: Correlation coefficients for amplitude and period metrics. 


\begin{tabular}{llr} 
Metric & Device & Effect Size \\
\hline \hline Mean trough period & Finger & 0.843 \\
Mean trough period & Foot & 0.422 \\
St.Dev. trough period & Finger & 1.128 \\
St.Dev. trough period & Foot & 0.221 \\
Mean cycle amplitudes & Finger & -0.520 \\
Mean cycle amplitudes & Foot & -0.699 \\
St.Dev. cycle amplitudes & Finger & 0.320 \\
St.Dev. cycle amplitudes & Foot & 0.065 \\
Number of taps & Finger & -0.757 \\
Number of taps & Foot & -0.577 \\
\hline
\end{tabular}

Table 15: On versus off effect size for amplitude and period metrics.

\subsubsection{Event Duration Metric Data}

The four conditions in Table 12 are computed for each of the six metrics in Table

11. Correlation coefficients with respect to the motor UPDRS are reported in Table

16, and effect sizes in Table 17. Select plots are presented in Chapter 6.2. 


\begin{tabular}{llcrr} 
Metric & Device & Med State & p value & Correlation \\
\hline \hline Total peak time & Finger & on & 0.804 & -0.055 \\
Total peak time & Foot & on & 0.486 & 0.153 \\
Total peak time & Finger & off & 0.421 & 0.176 \\
Total peak time & Foot & off & 0.094 & 0.357 \\
Total trough time & Finger & on & 0.808 & -0.054 \\
Total trough time & Foot & on & 0.569 & 0.125 \\
Total trough time & Finger & off & 0.287 & 0.232 \\
Total trough time & Foot & off & 0.316 & 0.219 \\
Total right saddle time & Finger & on & 0.941 & 0.016 \\
Total right saddle time & Foot & on & 0.714 & 0.081 \\
Total right saddle time & Finger & off & 0.625 & 0.108 \\
Total right saddle time & Foot & off & 0.412 & -0.180 \\
Total left saddle time & Finger & on & 0.961 & -0.011 \\
Total left saddle time & Foot & on & 0.704 & 0.084 \\
Total left saddle time & Finger & off & 0.132 & -0.324 \\
Total left saddle time & Foot & off & 0.009 & 0.535 \\
Total high trough time & Finger & on & 0.278 & -0.236 \\
Total high trough time & Foot & on & 0.137 & 0.319 \\
Total high trough time & Finger & off & 0.245 & -0.252 \\
Total high trough time & Foot & off & 0.481 & -0.155 \\
Total indeterminate event time & Finger & on & 0.044 & -0.424 \\
Total indeterminate event time & Foot & on & 0.108 & 0.344 \\
Total indeterminate event time & Finger & off & 0.157 & -0.305 \\
Total indeterminate event time & Foot & off & 0.814 & -0.052 \\
\hline
\end{tabular}

Table 16: Correlation coefficients for event duration metrics.

\begin{tabular}{llr} 
Metric & Device & Effect Size \\
\hline \hline Total peak time & Finger & 0.067 \\
Total peak time & Foot & -0.286 \\
Total trough time & Finger & -0.106 \\
Total trough time & Foot & 0.588 \\
Total right saddle time & Finger & -0.079 \\
Total right saddle time & Foot & -0.095 \\
Total left saddle time & Finger & 0.678 \\
Total left saddle time & Foot & 0.066 \\
Total high trough time & Finger & 0.014 \\
Total high trough time & Foot & -0.458 \\
Total indeterminate event time & Finger & 0.053 \\
Total indeterminate event time & Foot & 0.216 \\
\hline
\end{tabular}

Table 17: On versus off effect size of event duration metrics. 


\subsection{Discussion}

\subsubsection{Clinical Notes and Test Irregularities}

In addition to the annotations field on the data structure, clinicians were encouraged to provide notes on test sessions when they found this to be appropriate. A selection relevant to data exclusion and overall design are presented here. Subjects were identified by subject ID numbers assigned by the test administrators. Subjects were numbered 212 through 237.

- Subjects were tested on the side they reported as most affected, with the exception of subjects 221 and 224. Subject 224 tested on the left side after an unspecified failure of the right finger tapper, no explanation for subject 221 testing on the less affected side is available.

- The finger cap on the finger tapper came loose during subject 223's off state testing. The cap was repaired and the test was repeated.After inspection of the data, the subject was included in the analysis.

- Subject 230 did not receive a UPDRS rating in the off state, and is therefore excluded.

- The software crashed during subject 235's tapping test. As it was unclear exactly how to include a partial data set, the subject was excluded. 
- Subject 236 apparently dozed off during the tapping test. As it was unclear exactly how to account for this, the subject was excluded.

- The encoder apparently failed during one of subject 237's tapping tests. As it was unclear exactly how to include a partial data set, the subject was excluded.

- Of the 264 trials analyzed, 78 had average trough amplitudes over 100 degrees, implying they either experienced an odd software malfunction, or more likely the position was not initialized. Trials inspected visually had no other obvious irregularities, and consecutive trials were consistently offset. As the detection algorithm is robust to the offset, the trials were included. This affects 6 of 22 subjects.

\subsubsection{Amplitude, Period, and Event Duration Metrics}

The computation of the Mean Trough Period, Standard Deviation of Trough Period, Mean Cycle Amplitude, Standard Deviation of Cycle Amplitude, and Number of Taps is discussed in Section 5.3.1. Other researchers have found metrics related to rates of tapping [16] [33] [39] and amplitude of tapping [32] to correlate with the UPDRS. The UPDRS rating of the finger tapping task is based on a number of subjective criteria, including rate, amplitude, regularity and so forth. The analysis presented here considers only individual measurements in isolation. A more sophisticated analysis could combine the best metrics to produce a better assessment. 
Table 18 shows all metrics with correlation coefficients over 0.3 . The four highest correlations in the data presented are event duration metrics, associated with hesitations halts, or episodes of freezing. Left saddle time appears twice in the top four. This sort of feature would occur on the way up during a tapping task, suggesting hesitation. Figure 14 shows a time domain plot of a subject with an obvious hesitation at about 5.5 seconds. Note that the finger rests against the thumb at the lowest angle, in this case -80 degrees, where small hesitations are visible throughout. Plots of the left saddle means with respect to motor UPDRS are seen in Figures 15 and 20.

The presence of indeterminate event time as the metric with the second highest UPDRS correlation is surprising. Plots of this metric versus motor UPDRS are seen in Figures 16 and 19. It was originally included in the analysis mainly as a check on the event detection algorithm, and represents events not otherwise classified by the detection algorithm. Although these events are unclassified, an inspection of their occurrence suggests they are not entirely irrelevant to bradykinesia. The first and last events in a trial are by nature indeterminate, thus a large indeterminate score may indicate slow start, or inability to initialize movement. The second condition that produces an indeterminate detection is two events of the same amplitude, separated by a movement that does not trigger an event detection, as seen in Figure 13. An example might be a subject holding their foot down and twitching slightly, then returning to the same position. An excursion of less than $10 \mathrm{~ms}$ would not trigger a 
peak detection. This type of movement might again be associated with inability to efficiently initiate movement, hesitation or even tremor.
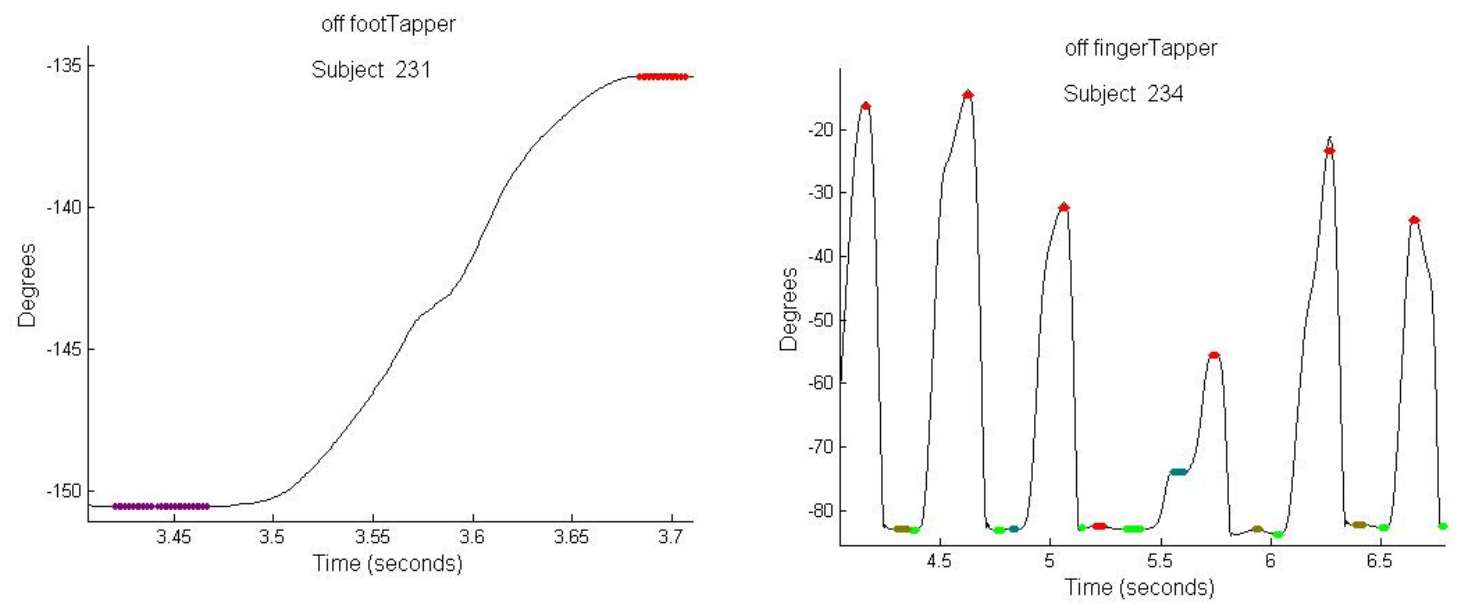

Figure 13: Detail of a trial showing a "split" Figure 14: Detail of a trial showing numerous hestrough, detected as indeterminate. itations and halts.

The best amplitude or duration metric is mean trough period, seen in Figure 18. The fact that it occurs with the foot tapper is encouraging, as foot tapping is underrepresented in the literature.

\begin{tabular}{llcrr} 
Metric & Device & Motor State & p Value & Correlation \\
\hline \hline Total left saddle time & Foot & off & 0.009 & 0.535 \\
Total indeterminate event time & Finger & on & 0.044 & -0.424 \\
Total peak time & Foot & off & 0.094 & 0.357 \\
Total left saddle time & Finger & off & 0.132 & -0.324 \\
Mean trough period & Foot & off & 0.108 & -0.344 \\
Total indeterminate event time & Foot & on & 0.108 & 0.344 \\
std trough period & Finger & off & 0.111 & 0.341 \\
Mean cycle amplitude & Foot & off & 0.121 & -0.333 \\
std cycle amplitude & Finger & off & 0.128 & 0.327 \\
Mean cycle amplitude & Finger & off & 0.152 & -0.309 \\
Total indeterminate event time & Finger & off & 0.157 & -0.305 \\
\hline
\end{tabular}

Table 18: Event duration and period metrics with highest correlation coefficients. 

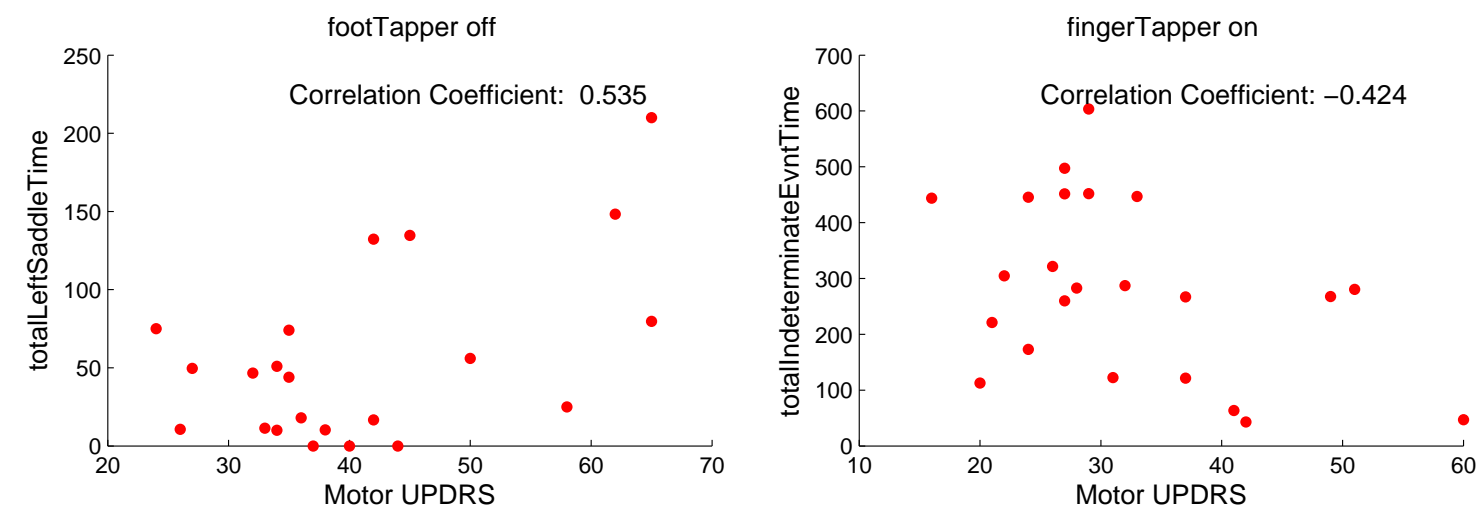

Figure 15: Plot of the correlation of left saddle Figure 16: Plot of the correlation of indeterminate time with respect to motor UPDRS. event time with respect to motor UPDRS
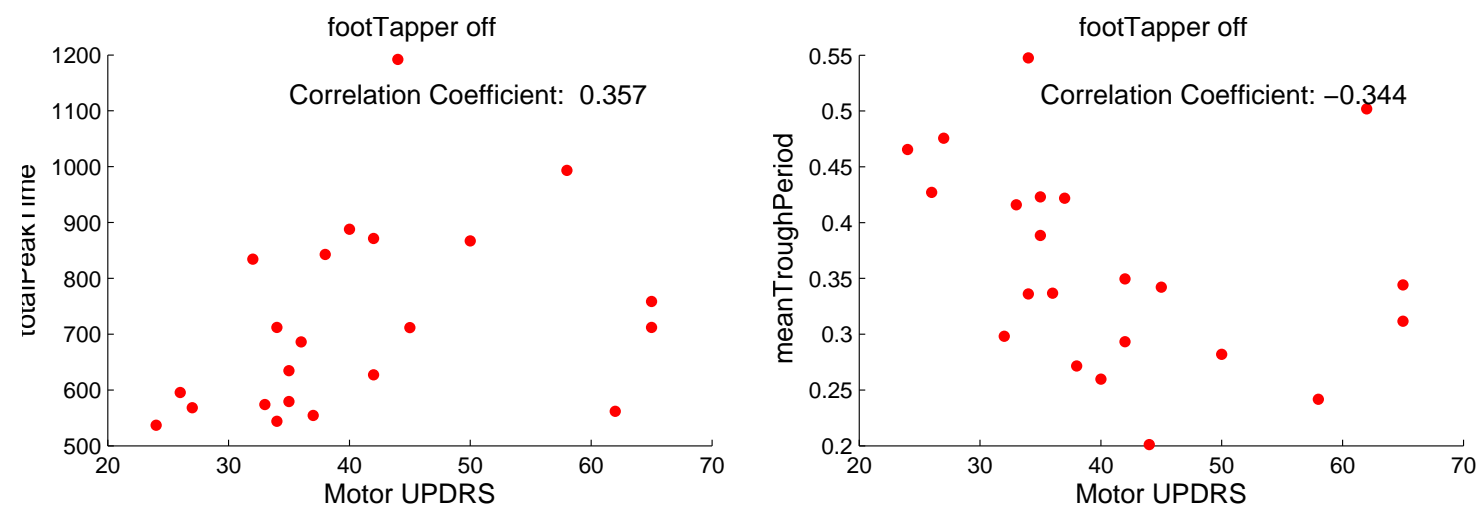

Figure 17: Plot of the correlation of total peak Figure 18: Plot of the correlation of mean trough time with respect to motor UPDRS. period with respect to motor UPDRS. 

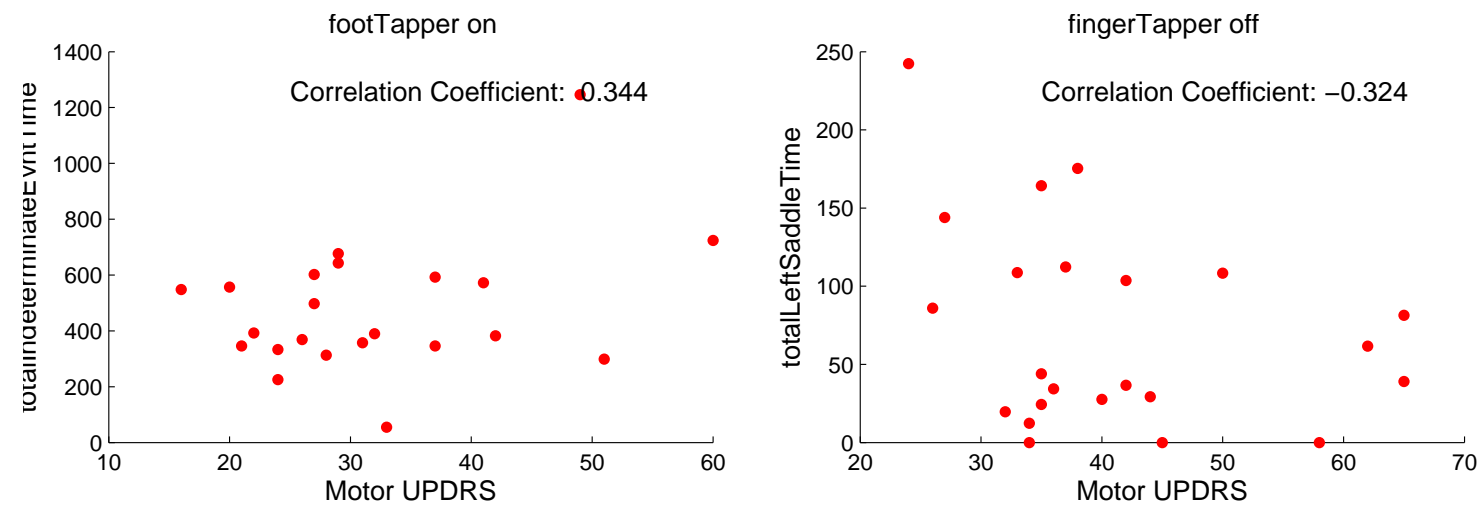

Figure 19: Plot of the correlation of indeterminate Figure 20: Plot of the correlation of left saddle event time with respect to motor UPDRS. time with respect to motor UPDRS 


\subsubsection{Effect Sizes}

To find the relationship between metrics and medication state, the computation and interpretation of effect size is described in Section 5.6. The equation for Cohen's $d$ is repeated here for convenience:

$$
d=\frac{\mu_{\mathrm{off}}-\mu_{\mathrm{on}}}{\frac{1}{2}\left(\sigma_{\mathrm{off}}+\sigma_{\mathrm{on}}\right)}
$$

All of the effect sizes over .3 are reported in Table 19, below. The level of .3 is chosen to exclude small effects.

\begin{tabular}{llr} 
Metric & Device & Effect Size \\
\hline \hline std trough period & finger tapper & 1.128 \\
mean trough period & finger tapper & 0.843 \\
number of taps & finger tapper & -0.757 \\
mean cycle amplitudes & foot tapper & -0.699 \\
total left saddle time & finger tapper & 0.678 \\
total trough time & foot tapper & 0.588 \\
number of taps & foot tapper & -0.577 \\
mean cycle amplitudes & finger tapper & -0.520 \\
total high trough time & foot tapper & -0.458 \\
std cycle amplitudes & finger tapper & 0.320 \\
\hline
\end{tabular}

Table 19: Effect size metrics with highest mean effect size.

The first notable thing about these values is the very large effect size of the leading metric. The mean effect size for the finger tapper standard deviation of trough period is greater than 1, indicating a strong effect. It also appears in Table 18. Although the correlation of .341 is not spectacular, it is the seventh highest presented here. This metric is similar to the variation in tapping examined by the button tests in 
Section 2.1.1 and repetitive finger tapping in Section 2.1.5. Since this has been shown to be effective by other researchers, it is not surprising that it is among the best metrics.

In contrast to the correlation values in Table 18 , four of the top five values in this table are finger tapper metrics, and none of the top four is an event duration metric. This is disappointing in that the effect size and UPDRS correlation do not seem to reinforce one another, with the exception of total left saddle time which appears on both tables. Mean cycle amplitude, standard deviation of cycle amplitude also appear on both Table 18 and Table 19, but UPDRS correlation coefficients are small for both.

A histogram of the effect size of the standard deviation of trough period for the finger tapper is plotted in Figure 21. The vertical line shows the mean value of all subjects. A mean that is shifted away from zero indicates a strong effect, and a preponderance of subjects on one side of zero supports that statement. In this case there are outliers present which probably shift the mean unduly to the right, but the bulk of subjects are on the same side of zero. This plot supports continued investigation of this metric.

Figure 22 shows a histogram of the effect size on mean trough period for the finger tapper. The vertical line is again the mean value of effect size for all trials. In this case the mean is shifted, and the bulk of the distribution is to the right of 

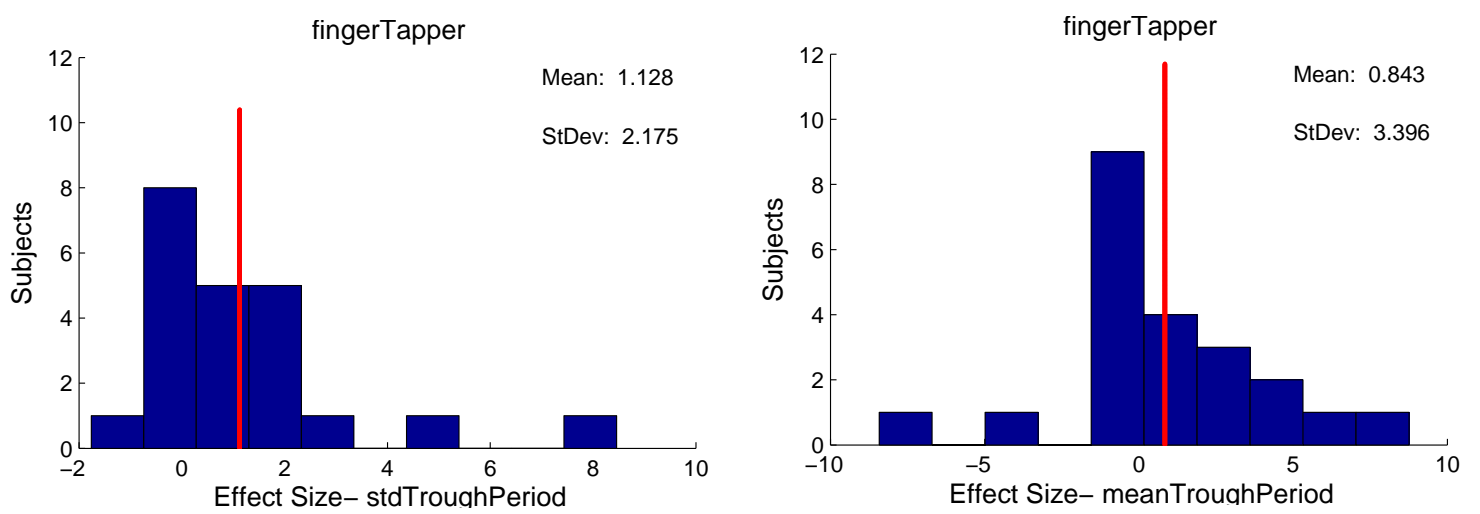

Figure 21: Plot of the effect size of trough period Figure 22: Plot of the effect size of mean trough standard deviation. period.

zero. Figures 23 and 24 are effect size histograms for number of taps and mean cycle amplitudes. The plot of the foot tapper in Figure 23 appears to be grouped around zero, with the mean shifted slightly negative. The outlier at ten is an unusually large effect size. The foot tapper in Figure 24 appears to have the mean shifted in to the left by negative outliers, and once again has a peak around zero.
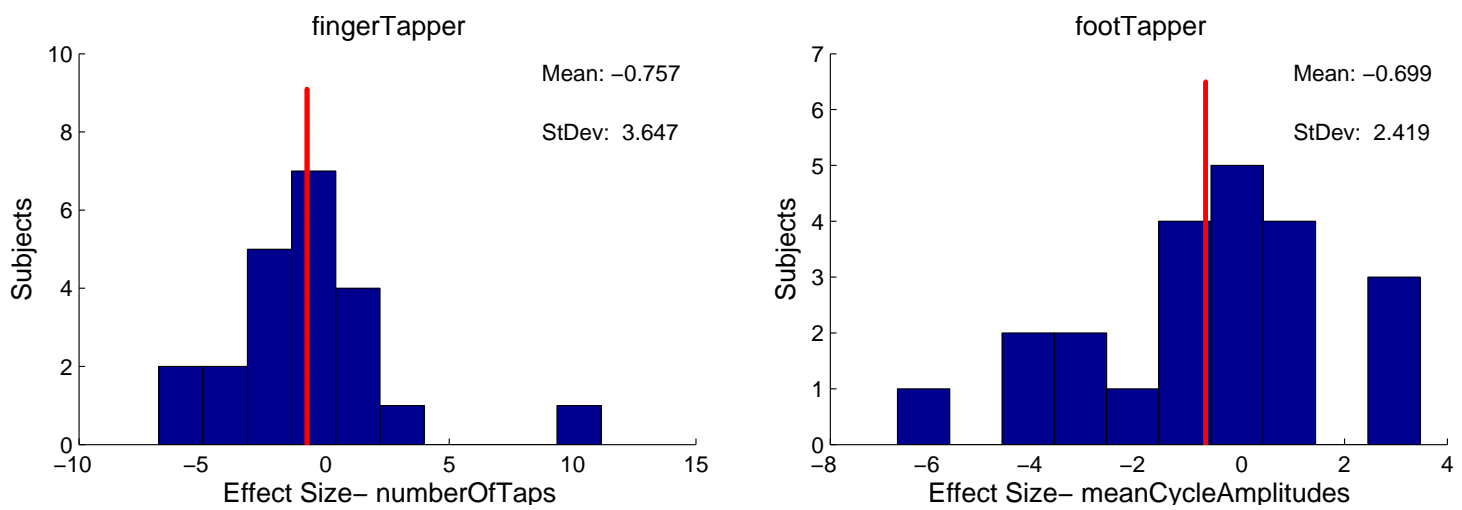

Figure 23: Plot of the effect size of the total num- Figure 24: Plot of the effect size of mean cycle ber of taps. amplitudes. 


\section{Summary \& Conclusion}

\subsection{Summary}

In this thesis two biomechanical devices were developed for the assessment of Parkinsonian bradykinesia. A literature survey was conducted on the state of the art of disease assessment, and requirements were developed. Durable prototypes were built and pilot data was collected in a clinical environment. Software was developed to administer tests and collect data. Algorithms and software were created to analyze data. The author is directly responsible for conduct of the literature survey, prototyping of several preliminary devices, specification and sourcing of all encoders and related materials, and design and implementation of data analysis software.

This thesis describes the design of the devices and the functionality of the data acquisition software, as well as the overall system used for data collection. A data analysis algorithm is fully described, and descriptive statistics of pilot data are reported. These statistics show promising correlations of time duration metrics with the motor subsection of the UPDRS, as well as good responsiveness to dopaminergic intervention. 


\subsection{Conclusion}

The high effect size of the finger tapper standard deviation of trough periods makes it a promising metric in terms of differentiation between medication states. Although correlation with the UPDRS is not high for any metrics, the results for event duration metrics were encouraging, suggesting that there might be a value in assessing halts and freezing. This, in turn, supports the measurement of full range of movement in assessment of Parkinsonian symptoms.

The devices described in this thesis are capable of making the same measurements examined by other researchers with button tests and finger tapping tests, with the addition of measuring the full range of motion. The resulting time duration metrics cannot be obtained from previously described devices such as keyboard finger tappers, button tests or pegboard tests. Furthermore, unlike full motion detection systems, these devices are inexpensive and suitable for use in the home.

The metric analysis does not clearly support either the finger or foot tapper to be significantly more responsive than the other. This supports their continued deployment as a battery, and possible future combination of metrics.

\subsection{Avenues for Further Research}

The devices presented in this thesis are part of an ongoing effort in the Biomedical Signal Processing Laboratory to develop a battery of test devices to augment and 
eventually replace the motor UPDRS. Due to the continuous nature of the design process, changes have already taken place that partially outdate the information presented here.

- The data analysis software is undergoing steady improvement. The detection algorithm could be improved to classify indeterminate events and provide better partial tap metrics, which might provide better assessments of hesitation and freezing.

- More powerful statistical methods will be introduced to form combinatorial metrics which might better separate medication states and have better correlation to the motor UPDRS.

- Updated prototype mechanical devices are being fabricated. Experiments with the manipulandum and finger cup on the finger tapper are ongoing. Future versions will be less restrictive and allow for measurement of both OHSU and UPDRS tapping. The next version of the foot tapper includes a goniometer/legbrace which standardizes the angle of the subject's leg, and may increase the repeatability of the device.

- The data acquisition software has been updated with a more pleasing appearance and a simpler controls. The automatic upload of data to a data server is near completion. 
- It is hoped that the data presented here will be augmented by analysis of additional data sets produced during the same clinical sessions. The value of the standard deviation metric in this thesis suggests that tests focused on pacing and variation in rate of tapping will likely be valuable. 


\section{References}

[1] Mittel C, Parkinson's Disease: Overview and Current Abstracts. Nova Science Publishers New York, 2003.

[2] Weiner W, Shulman L, Lang A. Parkinson's Disease: A complete Guide for Patients and Families. Johns Hopkins University Press, Baltimore MD, 2001.

[3] Huse D, Schulman K, Orsini L, Castelli-Haley J, Kennedy S, Lenhart G. Burden of Illness in Parkinson's Disease. Movement Disorders 2005; 20:1449-1454.

[4] Noyes K, Liu H, Holloway R, Dick A. Economic Burden Associated With Parkinson's Disease on Elderly Medicare Beneficiaries. Movement Disorders 2005; 21:362-372.

[5] Nutt J, Wooten G. Clinical Practice: Diagnosis and Initial Management of Parkinson's Disease. New England Journal Of Medicine, 2005; 353:1021-1027.

[6] Chrischilles E, Rubenstein L, Voelker M, Wallace R, Rodnitky R. The Health Burdens of Parkinson's Disease. Movement Disorders 1998; 13:406-413.

[7] Thobois S, Guillouet S, Broussolle E. Contributions of PET and SPECT to the Understanding of the Pathophysiology of Parkinson's Disease. Neurophysiol Clin 2001; 31:321-340. 
[8] Becker G, Muller A, Braune S, Buttner, T, Benecke R, Greulich W, Klien W, Mark G, Rieke J. Early Diagnosis of Parkinson's Disease. J Neurol 2002; $249: \mathrm{III} / 40-\mathrm{III} / 48$.

[9] Buddenberg L, Davis C. Test-Rest Relaibility of the Purdue Pegboard Test. American Journal of Occupational Therapy 2000; 54:555-558.

[10] Reddon J, Gill D, Gauk S, Maerz M. Purdue Pegboard: Test-Retest Estimates. Perceptual and Motor Skills 1988; 66:503-506.

[11] Haaxma C, Bloem B, Borm G, Horstink M. Comparison of a timed motor test battery to the Unified Parkinson's Disease Rating Scale-III in Parkinson's disease. Movement Disorders 2008; 23:1707-1717.

[12] Kandori A, Miyashita T, Hosono N, Yokoe M, Ogata K, Abe K, Sakoda S . Motion analysis of grip and release with fingers using simple magnetic detection system. Review of Scientific Instruments 2007; 78:034302-1-6.

[13] Espay A, Beaton D, Morgante F, Gunraj C, Lang A, Chen R. Impairments of speed and amplitude of movement in Parkinson's disease: a pilot study. Movement Disorders 2009; 24:1001-1008.

[14] Spyers-Ashby J, Stokes M, Bain P, Roberts S.Classification of normal and pathological tremors using a multidimensional electromagnetic system. Medical Engineering and Physics 1999; 21:713-723. 
[15] Liu X, Carroll C, Wang S, Zajicek J, Bain P . Quantifying drug-induced dyskinesias in the arms using digitised spiral drawing tasks. Journal of Neuroscience Methods 2005; 144:47-52.

[16] Dunnewold RJW, Jacobi CE, Van Hilton JJ. Quantitative assessment of bradykinesia in patients with parkinson's disease. J Neuroscience Methods 1997; 74:107-112.

[17] Sauermann S, Standhardt H, Gerschlager W, Lanmuller H, Alesch F. Kinematic evaluation in Parkinson's disease using a hand-held position transducer and computerized signal analysis. Aca Neurochirurgica 2005; 147:939-945.

[18] Ide J, Sugi T, Murakami N, Shima F, Shibasaki H, Nakamura M.. Quantitative assessment of hand movement on visual target tracking for patients with parkinson's disease. IEEE/ICME Int Conf on Complex Medical Eng 2007; 1896-1900. (Check Citation)

[19] Ghika J, Wiegner A, Fang JJ, Davies L, Young R, Growdon J. Portable system for quantifying motor abnormality in parkinson's disease. IEEE Trans on Med Eng; 40:276-282.

[20] Allen DP, Playfer JR, Aly NM, Duffey P, Heald A, Smith SL, Halliday DM. On the use of low cost peripherals for the assessment of motor dysfunction in 
parkinson's disease- quantification of bradykinesia using target tracking tasks. IEEE Trans on Neural Systems and Rehabilitation Eng 2007; 15:286-294 .

[21] Lin CH, Sullivan K, Wu A, Kantak S, Winstein C. Effect of task practice order on motor skill learning in adults with Parkinson disease: a pilot study. Physical Therapy 2007; 87:1120-1132. (Check Page Citation)

[22] Koop MM, Shivitz N, Bronte-Stewart H. Quantitative measures of fine motor, limb, and postural bradykinesia in very early stage, untreated parkinson's disease. Movement Disorders 2008; 23:1262-1268.

[23] Giovannoni G, Van Schalkwyk J, Fritz VU, Lees AJ. Bradykinesia akinesia inco-ordination test (BRAIN test): an objective computerized assessment of upper limb motor function. J Neurol Neurosurg Psychiatry 1999; 67:624-629.

[24] Ruiz P, Bernardos V. Evaluation of ActiTrac (ambulatory activity monitor) in parkinson's disease. J Neural Science 2008; 270:67-69.

[25] Papapetropoulos S, Jagid JR, Sengun C, Singer C, Gallo BV. Objective monitoring of tremor and bradykinesia during DBS surgery for parkinson disease. Neurology 2008; 70:1244-1249.

[26] Sherrill D, Hughes R, Salles S, Lie-Nemeth T, Akay M, Standaert D, Bonato P. Advanced analysis of wearable sensor data to adjust medication intake in 
parkinson's disease. Proc of 2nd Int IEEE EMBS Conf on Neural Eng; 5-8. (Check Citation)

[27] Andria G, Attivissimo F, Giaquinto N, Lanzolla A, Quagliarella L, Sasanelli N. Functional evaluation of handgrip signals for parkinsonian patients. IEEE trans on Inst and Measurement 2006; 55:1467-1473.

[28] Ward C, Sanes J, Dambrosia J, Calne D. Methods for evaluating treatment in parkinson's disease. Advances in Neurol 1983; 37:1-7.

[29] Myers LJ, MacKinnon. Quantification of movement regularity during internally and externally cued repetitive movements in patients with parkinson's disease. Proc of 2nd Int IEEE EMBS Conf on Neural Eng; 5-8. (Check Citation)

[30] Teasdale N, Phillips J, Stelmach. Temporal movement control in patients with parkinson's disease. J Neurol Neurosurg Psychiatry 1990; 53:862-868.

[31] Bonfiglioli C, DeBerti G, Nichelli P, Nicoletti R, Castiello U. Kinematic analysis of the reach to grasp movement in parkinson's and huntington's disease subjects. Neuropsychologia 1998; 36:1203-1208.

[32] Agostino R, Berardelli A, Curra A, Accornero N, Manfredi M. Clinical impairment of sequential finger movements in parkinson's disease. Movement Disorder 1998; 13:418-421. 
[33] Tavares A, Jeffries G, Koop M, Hill B, Hastie T, Heit G, Bronte-Stewart H. Quantitative measurements of alternating finger tapping in parkinson's disease correlate with UPDRS motor disability and reveal the improvement in fine motor control from medication and deep brain stimulation. Movement Disorders 2005; 20:1286-1298.

[34] Bronte-Stewart H, Ding L, Alexander C, Zhou Y, Moore, G. Quantitative digitography (QDG): a sensitive measure f digital motor control in idiopathic parkinson's disease. Movement Disorders 2000; 15:36-47.

[35] Ricards M, Marder K, Cote L, Mayeux R. Interrater reliability of the unified parkinson's disease rating scale motor examination. Movement Disorders 1994; 9:89-91.

[36] Curra A, Berardelli A, Agostino A, Modugno N, Puorger C, Accornero N, Manfredi M. Performance of sequential arm movements with and without advance knowledge of motor pathways in parkinson's disease. Movement Disorders 1997; 12:646-654.

[37] Gauntlett-Gilbert J, Brown V J, Reaction Time Deficits and Parkinson's. Neuroscience and Biobehavioral Review1998; 22:865-881.

[38] Nutt J G, Lea E S, Van Houten L, Schuff R A Sexton G J, Determinants of Tapping Speed in Normal Control Subjects and Subjects With Parkinson's 
Disease: Differing Effects of Brief and Continued Practice. Movement Disorders 2000; 15:843-849.

[39] Jobbagy A, Harcos P, Karoly R, Fazekas G, Analysis of Finger Tapping Movement. Journal of Neuroscience Methods 2005; 141:29-39.

[40] Montgomery E, Koller W, LaMantia T, Newman M, Swanson-Hyland E, Kaszniak A, Lyons K . Early detection of probable idiopathic Parkinson's disease:Development of a diagnostic test battery.Movement Disorders 2000; 15:467-473.

[41] Valentine J C, Cooper H. Effect size substantive interpretation guidelines: Issues in the interpretation of effect sizes. What Works Clearinghouse 2003; 